\title{
ANALYTIC OPERATOR ALGEBRAS (FACTORIZATION AND AN EXPECTATION)
}

BY

BARUCH SOLEL

\begin{abstract}
Let $M$ be a $\sigma$-finite von Neumann algebra and $\left\{\alpha_{t}\right\}_{t \in \mathbf{T}}$ a periodic flow on $M$. The algebra of analytic operators in $M$ is $\left\{a \in M: \operatorname{sp}_{\alpha}(a) \subseteq \mathbf{Z}_{+}\right\}$ and is denoted $H^{\infty}(\alpha)$. We prove that every invertible operator $a \in H^{\infty}(\alpha)$ can be written as $a=u b$, where $u$ is unitary in $M$ and $b \in H^{\infty}(\alpha) \cap H^{\infty}(\alpha)^{-1}$. We also prove inner-outer factorization results for $a \in H^{\infty}(\alpha)$.

Another result represents $H^{\infty}(\alpha)$ as the image of a certain nest subalgebra (of a von Neumann algebra that contains $M$ ) via a conditional expectation. As corollaries we prove a distance formula and an interpolation result for the case where $M$ is an injective von Neumann algebra.
\end{abstract}

1. Introduction. In this paper we intend to study some aspects of analyticity in operator algebras. In [1] W. Arveson presented the theory of subdiagonal algebras as a noncommutative analogoue of the theory of weak* Dirichlet algebras.

In [6] R. Loebl and P. Muhly have shown that some subdiagonal algebras arise as algebras of analytic operators with respect to a flow on a von Neumann algebras. Similar results were obtained independently by S. Kawamura and J. Tomiyama in [5]. Let $M$ be a von Neumann algebra and $\left\{\alpha_{t}\right\}_{t \in \mathbf{R}}$ a $\sigma$-weakly continuous representation of $\mathbf{R}$ as *-automorphisms of $M$ (i.e. $\alpha$ is a flow on $M$ ). Let $H^{\infty}(\alpha)$ be the set $\left\{a \in M: \operatorname{sp}_{\alpha}(a) \subseteq[0, \infty)\right\}$, where $\operatorname{sp}_{\alpha}(a)$ is Arveson's spectrum of $a$ with respect to $\alpha$.

It was shown in [6] that $H^{\infty}(\alpha)$ is an algebra and if $M$ is $\mathbf{R}$-finite, then $H^{\infty}(\alpha)$ is a maximal subdiagonal algebra. This class of algebras, obtained in this manner from flows on von Neumann algebras, contains the analytic crossed products (also called nonsefladjoint crossed products) and the nest subalgebras (see [6] for details). The structure of these algebras $H^{\infty}(\alpha)$ was further studied by several authors (see [7-15]).

In this paper we continue the study of the algebras $H^{\infty}(\alpha)$ with the assumption that the flow $\alpha$ is periodic and $M$ is a $\sigma$-finite von Neumann algebra.

The main result of $\S 3$ is Theorem 3.10 which shows that every invertible operator $a \in M$ can be written as $a=u b$, where $u$ is unitary in $M$ and $B \in H^{\infty}(\alpha) \cap$ $H^{\infty}(\alpha)^{-1}$. This result was proved in $[\mathbf{1}]$ for finite maximal subdiagonal algebras. We, however, do not assume that $M$ is finite. The result is also related to a factorization result of an operator with respect to a nest algebra (see $[3$, Theorem 3.3]) and we will comment on this later (see the discussion following Proposition 3.7).

Received by the editors February 7, 1984.

1980 Mathematics Subject Classification. Primary 46L10, 47D25.

Key words and phrases. Flow, analytic operators with respect to a flow, inner-outer factorization, expectation, distance estimate, interpolation. 
On the way to the main result we prove some factorization results for vectors in $H$ (where $M$ is represented on a Hilbert space $H$ with a separating and cyclic vector $\xi$ such that $a \rightarrow\langle a \xi, \xi\rangle$ is an $\alpha$-invariant state on $M)$ that extend the classical inner-outer factorization (when $M=L^{\infty}(\mathbf{T})$ and $\alpha$ acts by translation, $H^{\infty}(\alpha)$ is the classical Hardy space $\left.H^{\infty}(\mathbf{T})\right)$. This is also used to obtain results on subspaces of $H$ that are invariant under $H^{\infty}(\alpha)$ and are generated by a single vector in $H$.

In $\S 4$ we introduce an expectation from a certain von Neumann algebra $R_{0}^{\prime}$ onto $M$ such that it maps a certain nest subalgebra of $R_{0}^{\prime}$ onto $H^{\infty}(\alpha)$. If $H=L^{2}(\mathbf{T})$, $M=L^{\infty}(\mathbf{T})$ and $H^{\infty}(\alpha)=H^{\infty}(\mathbf{T})$, then $R_{0}^{\prime}=B(H)$ (the algebra of all bounded operators on $H$ ) and this expectation is the one used by Arveson in [3, §5]. We believe that this expectation might help to "carry" results that can be proved for nest algebras, or nest subalgebras (of von Neumann algebras) to the algebra $H^{\infty}(\alpha)$. As examples we prove a distance formula (Corollary 4.7) and an interpolation result (Corollary 4.8).

2. Preliminaries. Let $M$ be a $\sigma$-finite von Neumann algebra acting on a Hilbert space $H$ and let $\left\{\alpha_{t}\right\}_{t \in \mathbf{R}}$ be a periodic flow (i.e. a periodic $\sigma$-weakly continuous representation of $\mathbf{R}$ as *automorphisms of $M$ ). We assume that the period is $2 \pi$ and write $\mathbf{T}$ for the interval $[0,2 \pi]$ identified with the unit circle. Since $\mathbf{T}$ is compact, the map $\varepsilon_{0}$, defined by $\varepsilon_{0}(x)=\int_{0}^{2 \pi} \alpha_{t}(x) d t, x \in M$, is a well-defined, linear, $\sigma$-weakly continuous map from $M$ onto the fixed point algebra, $M_{0}$, of $M$ with respect to $\left\{\alpha_{t}\right\}$ (dt denotes the normalized Lebesgue measure on $\mathbf{T}$ ). In fact, $\varepsilon_{0}$ is a faithful normal expectation from $M$ onto $M_{0}$ such that $\varepsilon_{0} \circ \alpha_{t}=\varepsilon_{0}, t \in \mathbf{T}$. By choosing a faithful normal state $\phi_{0}$ of $M_{0}$ and letting $\phi$ be $\phi_{0} \circ \varepsilon_{0}$ we get a faithful normal state $\phi$ on $M$ that is $\left\{\alpha_{t}\right\}$-invariant (i.e. $\phi \circ \alpha_{t}=\phi, t \in \mathbf{T}$ ). Considering the Gelfand-Naimark-Segal construction of $\phi$, we may suppose that $M$ has a separating and cyclic vector $\xi \in H$ such that $\phi(x)=\langle x \xi, \xi\rangle$ is an $\left\{\alpha_{t}\right\}$-invariant state on $M$. The algebra $M$, the flow $\alpha$, the space $H$ and the vector $\xi$, as above, will be fixed throughout the paper.

We now define a canonical pair to be a pair $\{B, \eta\}$ with the following properties:

(1) $B$ is a $\sigma$-finite von Neumann algebra acting on $H$.

(2) $\xi$ is a separating and cyclic vector for $B$.

(3) $\left\{\eta_{t}\right\}_{t \in \mathbf{T}}$ is a periodic flow on $B$.

(4) $\langle a \xi, \xi\rangle=\left\langle\eta_{t}(a) \xi, \xi\right\rangle$ for $t \in \mathbf{T}, a \in B$.

The discussion above shows that $\{M, \alpha\}$ is a canonical pair. Property (4) (applied to $\{M, \alpha\})$ shows that we can define unitary operators $\left\{W_{t}: t \in \mathbf{T}\right\}$ such that $W_{t} a \xi=\alpha_{t}(a) \xi, t \in \mathbf{T}, a \in M$. Let us now write $R$ for the commutant of $M$ and let $\gamma$ be the flow on $R$ defined by $\gamma_{t}(a)=W_{t} a W_{t}^{*}, t \in \mathbf{T}, a \in R$. Then $\left\langle\gamma_{t}(a) \xi, \xi\right\rangle=\left\langle W_{t} a W_{t}^{*} \xi, \xi\right\rangle=\langle a \xi, \xi\rangle, a \in R$. Hence $\{R, \gamma\}$ is a canonical pair.

The two canonical pairs $\{M, \alpha\}$ and $\{R, \gamma\}$ will be fixed throughout the paper. In the remainder of this section we will describe some of the structure of a canonical pair and set up notation. Since we will be mostly interested in the pairs $\{M, \alpha\}$ and $\{R, \gamma\}$, we will introduce the notation with respect to $\{M, \alpha\}$ and in parentheses the analogous notation for $\{R, \gamma\}$. Of course, for all the results that will be introduced or defined for $\{M, \alpha\}$, there are analogous results for $\{R, \gamma\}$. 
For each $n \in \mathbf{Z}$ we define a $\sigma$-weakly continuous linear map $\varepsilon_{n}$ on $M$ (and analogously $\lambda_{n}$ for $R$ ) by

$$
\varepsilon_{n}(x)=\int_{0}^{2 \pi} e^{-i t n} \alpha_{t}(x) d t, \quad x \in M .
$$

Let $M_{n}$ be $\varepsilon_{n}(M)$ (and $R_{n}=\lambda_{n}(R)$ ). Then it is easy to check that $M_{n}=\{x \in$ $\left.M: \alpha_{t}(x)=e^{i n t} x, t \in \mathbf{T}\right\}$.

For each $n \in \mathbf{Z}$ define a projection $f_{n}$ by

$$
\begin{gathered}
f_{n}=\sup \left\{u u^{*}: u \text { is a partial isometry in } M_{n}\right\} \\
\left(g_{n}=\sup \left\{u u^{*}: u \text { is a partial isometry in } R_{n}\right\}\right) .
\end{gathered}
$$

Then, by $\left[\mathbf{1 2}\right.$, Lemma 2.2] the $f_{n}$ lie in $Z\left(M_{0}\right)$ (the centre of $\left.M_{0}\right)$. The following lemma appears in $[\mathbf{1 1}]$.

LEMMA 2.1. (1) For every $n, m \in \mathbf{Z}, M_{n} M_{m} \subseteq M_{n+m}, M_{n}^{*}=M_{-n}$.

(2) Let $x \in M_{n}$ and let $x=v|x|$ be the polar decomposition of $x$.

Then $v \in M_{n}$ and $|x| \in M_{0}$.

The following result can be found in [14, Proposition 2.3 and Theorem 2.4]. Although it was assumed there that the algebra $M$ is finite, this assumption was not used for the proof of this result.

Proposition 2.2. Fix $n \in \mathbf{Z}$. Then there is a sequence $\left\{v_{n, m}\right\}_{m=1}^{\infty}$ of partial isometries in $M_{n}$ with the following properties:

(1) $v_{n, m}^{*} v_{n, j}=0$ if $m \neq j$,

(2) $\sum_{m=1}^{\infty} v_{n, m} v_{n, m}^{*}=f_{n}$,

(3) $M_{n}=\sum_{m=1}^{\infty} v_{n, m} M_{0}$; i.e. every $x \in M_{n}$ can be written as $\sum_{m=1}^{\infty} v_{n, m} x_{m}$ for some $x_{m} \in M_{0}$, where the sum converges to the $\sigma$-weak operator topology. (For $\{R, \gamma\}$ we write $\left\{u_{n, m}\right\}_{m=1}^{\infty} \subseteq R_{n}$.)

For a flow $\eta$ on a von Neumann algebra $B$, let $B^{\eta}(S)$ denote the spectral subspace associated with $S$; i.e. $B^{\eta}(S)=\left\{x \in B: \operatorname{sp}_{\eta}(x) \subseteq S\right\}$.

We write $H^{\infty}(\alpha)$ for $M^{\alpha}(\{n \in \mathbf{Z}: n \geq 0\})$ and $H_{0}^{\infty}(\alpha)$ for $M^{\alpha}(\{n \in \mathbf{Z}: n>0\})$. (Similarly $H^{\infty}(\gamma)$ and $H_{0}^{\infty}(\gamma)$ are defined.)

We have, for $n \in \mathbf{Z}, M^{\alpha}(\{n\})=M_{n}$ and, for $x \in M, \operatorname{sp}_{\alpha}(x)=\left\{n \in \mathbf{Z}: \varepsilon_{n}(x) \neq\right.$ $0\}$. When it causes no confusion we write $H^{\infty}$ in place of $H^{\infty}(\alpha)$.

The following result appears in [12, Theorem 2.4].

Proposition 2.3. (1) $H^{\infty}(\alpha)=\left\{x \in M: \varepsilon_{n}(x)=0\right.$ for $\left.n<0\right\}$.

(2) $H^{\infty}(\alpha)$ is the $\sigma$-weakly closed subalgebra of $M$ generated by $M_{0}$ and all partial isometries in $\bigcup\left\{M_{n}: n>0\right\}$.

In fact, by [9, Theorem 1] $M$ is linearly spanned by $\bigcup\left\{M_{n}: n \in \mathbf{Z}\right\}$ in the $\sigma$-weak operator topology.

For a subset $S \subseteq H$ let $[S]$ denote the closed linear subspace spanned by $S$. For $m \neq n, a \in M_{n}, b \in M_{m}$, we have $\langle a \xi, b \xi\rangle=\left\langle\alpha_{t}\left(b^{*} a\right) \xi, \xi\right\rangle$ for each $t \in \mathbf{T}$ and therefore $\langle a \xi, b \xi\rangle=\left\langle\varepsilon_{0}\left(b^{*} a\right) \xi, \xi\right\rangle=0$. Hence $\left\{\left[M_{n} \xi\right]\right\}_{n \in \mathbf{Z}}$ is an orthogonal family of subspaces. Let $E_{n}$ be the projection onto $\left[M_{n} \xi\right]$. Then $E_{n} \in M_{0}^{\prime}$ and $\sum E_{n}=I$ (as $\xi$ is a cyclic vector for $M$ and $[M \xi]=\sum_{n} \bigoplus\left[M_{n} \xi\right]$ ). Note also that $W_{t}=\sum_{n=-\infty}^{\infty} e^{i n t} E_{n}$ is the spectral decomposition of $\left\{W_{t}\right\}_{t \in \mathbf{T}}$ (hence we also 
have that $E_{n}$ is the projection onto $\left.\left[R_{n} \xi\right]\right)$ and $E_{n} a \xi=\varepsilon_{n}(a) \xi$ for $n \in \mathbf{Z}, a \in M$. We write $H^{2}$ for $\sum_{n=0}^{\infty} \bigoplus\left[M_{n} \xi\right]$ and $H_{0}^{2}$ for $\sum_{n=1}^{\infty} \bigoplus\left[M_{n} \xi\right]$. For $n \in \mathbf{Z}$ we let $P_{n}$ be the orthogonal projection onto $\sum_{m=n}^{\infty} \bigoplus\left[M_{n} \xi\right]$; i.e. $P_{n}=\sum_{m=n}^{\infty} E_{m}$ (and $P_{n}$ is the projection onto $\left.\left[M^{\alpha}[n, \infty) \xi\right]=\left[R^{\gamma}[n, \infty) \xi\right]\right)$. Then $P_{n+1} \leq P_{n}$ for $n \in \mathbf{Z}$, $\bigvee_{n=-\infty}^{\infty} P_{n}=I$, and $\bigwedge_{n=-\infty}^{\infty} P_{n}=0$.

With the partial isometries $\left\{v_{n, m}: n \in \mathbf{Z}, m \geq 1\right\}$ defined as in Proposition 2.2, we can define maps $\left\{\beta_{n}\right\}_{n \in \mathbf{Z}}$ on $M_{0}^{\prime}$ by the formula

$$
\beta_{n}(T)=\sum_{m=1}^{\infty} v_{n, m} T v_{n, m}^{*}
$$

(and on $R_{0}^{\prime}, \eta_{n}(T)=\sum_{m=1}^{\infty} u_{n, m} T u_{n, m}^{*}$ ).

We summarize some of the properties of $\left\{\beta_{n}\right\}_{n \in \mathbf{Z}}$ that will be used later in the following (for its proof see [15, Lemma 2.4]).

LEMMA 2.4. Fix $n, m \in \mathbf{Z}$.

(1) $\beta_{n}$ is a well-defined ${ }^{*}$-homomorphism from $M_{0}^{\prime}$ onto $f_{n} M_{0}^{\prime}$ whose restriction to $f_{-n} M_{0}^{\prime}$ is $a{ }^{*}$-isomorphism onto $f_{n} M_{0}^{\prime}$.

(2) Suppose $Q \in M_{0}^{\prime}$ is a projection. Then

$$
\beta_{n}(Q)=\bigvee\left\{u Q u^{*}: u \in M_{n} \text { is a partial isometry }\right\} .
$$

(3) For $T \in M_{0}^{\prime}, \beta_{n} \beta_{m}(T)=f_{n} \beta_{n+m}(T)$.

(4) For $T \in M_{0}^{\prime}$, $T$ lies in $M^{\prime}(=R)$ if and only if $\beta_{j}(T)=f_{j} T$ for each $j \in \mathbf{Z}$.

(5) $\beta_{n}\left(E_{0}\right)=E_{n}$ (hence $\beta_{m}\left(E_{n}\right)=\beta_{m} \beta_{n}\left(E_{0}\right)=f_{n} E_{n+m}$ and, consequently, $\left.\beta_{m}\left(P_{n}\right)=f_{n} P_{n+m}\right)$.

The following notation and definitions will be used later:

1. A projection $Q \in M_{0}^{\prime}$ is said to be an $M$-wandering projection if, for each $n \in \mathbf{Z}, Q \beta_{n}(Q)=0$ (note that this implies that $\beta_{n}(Q) \beta_{m}(Q)=0$ for $n \neq m$ ). The set of all the $M$-wandering projections in $M_{0}^{\prime}$ will be denoted $P_{1}$.

2. For $Q \in \mathcal{P}_{1}$ we let $\sigma(Q)$ be $\sum_{n=0}^{\infty} \beta_{n}(Q)$ and we write $\mathcal{P}_{3}$ for $\sigma\left(P_{1}\right)$.

3. A closed subspace $\mathcal{M}$ of $H$ is called invariant if $a x \in \mathcal{M}$ for each $a \in H^{\infty}(\alpha)$ and $x \in \mathcal{M}$. Let us denote by $\mathcal{P}_{2}$ the set of all orthogonal projections whose range is an invariant subspace (as $M_{0} \subseteq H^{\infty}(\alpha), P_{2} \subseteq M_{0}^{\prime}$ ).

4. For $P \in P_{2}$ we let $\delta(P)$ be $P-\vee\left\{\beta_{n}(P): n>0\right\}$.

The following lemma was proved in $[\mathbf{1 4}]$.

LEMMA 2.5. If $P \in P_{2}$, then $\delta(P) \in P_{1}$ and

$$
P=\sigma(\delta(P))+\bigwedge_{n>0} \bigvee_{m \geq n} \beta_{m}(P) .
$$

The projection $\bigwedge_{n>0} \bigvee_{m \geq n} \beta_{m}(P)$ lies in $M^{\prime}$ and $P \in P_{3}$ iff $\bigwedge_{n>0} \bigvee_{m \geq n} \beta_{m}(P)=$ 0 .

Note that if $Q, P \in P_{2}$ and $Q \leq P \in P_{3}\left(=\sigma\left(P_{1}\right)\right)$ then $Q \in P_{3}$ since $\bigwedge_{n>0} \bigvee_{m \geq n} \beta_{n}(Q) \leq \bigwedge_{n>0} \bigvee_{m \geq n} \beta_{n}(P)=0$. In particular any $Q \in P_{2}$ that satisfies $Q \leq P_{0}$ (i.e. $Q(H)$ is an invariant subspace, contained in $H^{2}$ ) is in $P_{3}$.

LEMMA 2.6. $H^{\infty}(\alpha)=M \cap \operatorname{Alg}\left\{P_{n}: n \in \mathbf{Z}\right\}$; i.e. $a \in H^{\infty}(\alpha)$ if and only if $a \in M$ and $P_{n} a P_{n}=a P_{n}$ for each $n \in \mathbf{Z}$.

PROOF. Immediate from [6, Corollary 2.14]. 
LEMMA 2.7. Let $c\left(E_{n}\right)$ be the central support of $E_{n}$ (as a projection in $M_{0}^{\prime}$ ). Then $f_{r_{\iota}}=c\left(E_{n}\right)$.

Proof. Since $f_{n} \in Z\left(M_{0}\right)$ and $f_{n} E_{n}=E_{n}$ we have $f_{n} \geq c\left(E_{n}\right)$. Suppose $z$ is a nonzero projection in $Z\left(M_{0}\right)$ such that $z E_{n}=0$ and $z \leq f_{n}$. Then there is a nonzero partial isometry $v \in M_{n}$ such that $v v^{*} \leq z$. Since $z E_{n}=0$ and $v \xi \in E_{n}(H), z v \xi=0$ and $v v^{*} v \xi=0$. Hence $v \xi=0$. Since $\xi$ is a separating vector and $v \neq 0$ we get a contradiction. Therefore $f_{n}=c\left(E_{n}\right)$.

3. Factorization and invariant subspaces. For $g \in H$ let $\mathcal{M}(g)$ be $\left[H^{\infty} g\right]$ and $P(g)$ be the projection onto $\mathcal{M}(g)$. Then $P(g) \in \mathcal{P}_{2}$.

PROPOSITION 3.1. For $g \in H$ the following two conditions are equivalent:

(1) $P(g) \in P_{3}$.

(2) There is a partial isometry $U \in R$ such that $U^{*} g \in H^{2}$ and $U U^{*} g=g$.

Moreover,

(3) If (2) is satisfied, then we can choose $U$ in such a way that $U^{*} U \in R_{0}$ and $\mathcal{M}\left(U^{*} g\right)=\mathcal{M}\left(E_{0} U^{*} g\right)$.

(4) If (1) is satisfied and $P(g)=\sigma(Q), Q \in P_{1}$, then $\left[M_{0} Q g\right]=Q(H)$ and $\beta_{n}(Q)(H)=\left[M_{n} Q g\right]$ for $n \in \mathbf{Z}$.

ProOF. (2) implies (1). Suppose that $f=U^{*} g \in H^{2}$ and $U f=g$ for some partial isometry $U \in R$. Then $P(g)=U P(f) U^{*}$. But $P(g) \leq P_{0}$, hence $P(f) \in P_{3}$. Now if $P(f)=\sigma(Q), Q \in P_{1}$, then $P(g)=\sigma\left(U Q U^{*}\right)$ and $U Q U^{*} \in P_{1}$.

(1) implies (2). Suppose now that $P(g) \in P_{3}$, hence $P(g)=\sigma(\delta(P(g)))$, and let $Q$ be $\delta(P(g))$. Let $a$ be in $M_{n}$ for some $n>0$. Then $a=\sum v_{n, m} x_{m}$ for some $x_{m}$ in $M_{0}$ and $a Q g=\sum v_{n, m} Q x_{m} g \in \beta_{n}(Q)(H)$. Since $Q$ is $M$-wandering, $h=Q g$ is a wandering vector in the sense that $\langle a h, h\rangle=0$ for $a \in M$ with $\varepsilon_{0}(a)=0$. Hence $\langle a h, h\rangle=\left\langle\varepsilon_{0}(a) h, h\right\rangle$ for all $a \in M$. The map $a \rightarrow\langle a h, h\rangle$ is a normal positive linear functional on $M_{0}$. Consider $M_{0}$ as acting on $E_{0}(H)=\left[M_{0} \xi\right]$; then $\xi$ is a cyclic and separating vector for $M_{0}$ and thus (by [4, Theorem 4, Part III, Chapter 1]) there is some $h_{0} \in\left[M_{0} \xi\right]$ such that $\left\langle a h_{0}, h_{0}\right\rangle=\langle a h, h\rangle$ for every $a \in M_{0}$. For $a \in M_{n}$, $n \neq 0, a h_{0} \in E_{n}(H)$ and $\left\langle a h_{0}, h_{0}\right\rangle=0(=\langle a h, h\rangle)$. Hence $\left\langle a h_{0}, h_{0}\right\rangle=\langle a h, h\rangle$ for each $a \in M$.

Now define a partial isometry $U$ on $H$ by extending the isometric map $a h_{0} \rightarrow a h$, $a \in M$. Then the initial subspace of $U$ is $\left[M h_{0}\right]$ and the final subspace is $[M h]$.

Since, for $b \in M, U b a h_{0}=b a h=b U a h_{0}, U \in M^{\prime}=R$.

Since $W_{t} a h_{0}=\alpha_{t}(a) W_{t} h_{0}=\alpha_{t}(a) h_{0}$ for $a \in M, t \in \mathbf{T}, W_{t}\left[M h_{0}\right]=\left[\alpha_{t}(M) h_{0}\right]=$ $\left[M h_{0}\right]$ for each $t \in \mathbf{T}$. Hence $W_{t} U^{*} U W_{t}=U^{*} U$. Thus $U^{*} U \in R_{0}$. Let $f$ be $U^{*} g$.

It is left to show that, with this choice of $U$, we get $\mathcal{M}(f)=\mathcal{M}\left(E_{0} f\right)$. For this, note that we have:

(a)

$$
\left[M_{0}(1-Q) g\right]=(1-Q)\left[M_{0} g\right] \subseteq \sum_{n=1}^{\infty} \beta_{n}(Q)(H)
$$

(as $\left.\left[H^{\infty} g\right]=\sum_{n=0}^{\infty} \beta_{n}(Q)(H)\right)$.

(b) $\left[M_{0} Q g\right] \subseteq Q(H)$.

(c) $\left[H_{0} g\right] \subseteq \sum_{n=1}^{\infty} \beta_{n}(Q)(H)\left(\right.$ as $a \beta_{n}(Q) g \in \beta_{n+k}(Q)(H)$ for $\left.a \in M_{k}\right)$. 
This implies that $Q(H)=Q\left[H^{\infty} g\right]=Q\left[M_{0} g\right]=\left[M_{0} Q g\right]=\left[M_{0} h\right]$. Also, for $n \in \mathbf{Z},\left[M_{n} h\right]=V\left\{\left[v M_{0} h\right]: v \in M_{n}\right.$ is a partial isometry $\}=\beta_{n}(Q)(H)$. Hence $\left[H^{\infty} g\right]=\left[H^{\infty} h\right]$. But

$$
\mathcal{M}(f)=\left[H^{\infty} f\right]=\left[H^{\infty} U^{*} g\right]=U^{*}\left[H^{\infty} g\right]=U^{*}\left[H^{\infty} h\right]=\left[H^{\infty} h_{0}\right],
$$

and

$$
\begin{aligned}
f-h_{0} & =U^{*}(g-Q g)=U^{*}(1-Q) g \in U^{*} \sum_{n=1}^{\infty} \beta_{n}(Q)(H) \\
& =U^{*} \sum_{n=1}^{\infty} \bigoplus\left[M_{n} h\right]=\sum_{n=1}^{\infty} \bigoplus\left[M_{h} h_{0}\right] \subseteq \sum_{n=1}^{\infty} E_{n}(H) .
\end{aligned}
$$

Hence $h_{0}=E_{0} f$ and $\left[H^{\infty} f\right]=\left[H^{\infty} h_{0}\right]=\left[H^{\infty} E_{0} f\right]$.

The following proposition shows the uniqueness of the above representation.

Proposition 3.2. Suppose $g \in H$ and $g=U_{1} f^{1}=U_{2} f^{2}$ such that, for $i=1,2$,

(1) $U_{i}$ is a partial isometry in $R$ with initial subspace $\left[M f^{i}\right]$,

(2) $\left[H^{\infty} f^{i}\right]=\left[H^{\infty} E_{0} f^{i}\right] \subseteq H^{2}$.

Then $U_{2}^{*} U_{1}$ and $U_{1}^{*} U_{2}$ lie in $R_{0}$.

PROOF. Since $g$ has such a representation, $P(g)=\sum_{n=0}^{\infty} \beta_{n}(Q)$ for some $Q \in$ $P_{1}$. We first claim that $U_{i}^{*} Q g \in E_{0}(H), i=1,2$. Fix $i=1$ or 2 and let $h_{0}$ be $U_{i}^{*} Q g$. Then $h_{0}=U_{i}^{*} Q g \in U_{i}^{*}\left[H^{\infty} g\right]=\left[H^{\infty} f^{i}\right] \subseteq H^{2}$. Using Proposition 3.1(4) we see that $Q(H)=\left[M_{0} Q g\right], \beta_{n}(Q)(H)=\left[M_{n} Q g\right]$ for $n \in \mathbf{Z}$, and $\left[H^{\infty} g\right]=\sum_{n=0}^{\infty} \beta_{n}(Q)(H)=\left[H^{\infty} Q g\right]$. Hence $\left[H^{\infty} E_{0} f^{i}\right]=\left[H^{\infty} f^{i}\right]=U_{i}^{*}\left[H^{\infty} g\right]=$ $U_{i}^{*}\left[H^{\infty} Q g\right]=\left[H^{\infty} h_{0}\right]$ and consequently

$$
\left[H_{0}^{\infty} E_{0} f^{i}\right]=\left[H_{0}^{\infty}\left[H^{\infty} E_{0} f^{i}\right]\right]=\left[H_{0}^{\infty}\left[H^{\infty} h_{0}\right]\right]=\left[H_{0}^{\infty} h_{0}\right]
$$

We then have $\left[M_{0} h_{0}\right]=\left[M_{0} E_{0} f^{i}\right]$ since

$$
\left[H^{\infty} E_{0} f^{i}\right]=\left[M_{0} E_{0} f^{i}\right] \oplus\left[H_{0}^{\infty} E_{0} f^{i}\right]
$$

and

$$
\begin{aligned}
{\left[H^{\infty} h_{0}\right] } & =U_{i}^{*}\left[H^{\infty} Q g\right]=U_{i}^{*}\left(\left[H_{0}^{\infty} Q g\right] \oplus\left[M_{0} Q g\right]\right) \\
& =\left[H_{0}^{\infty} h_{0}\right] \oplus\left[M_{0} h_{0}\right]=\left[H_{0}^{\infty} E_{0} f^{i}\right] \oplus\left[M_{0} h_{0}\right] .
\end{aligned}
$$

In particular $h_{0} \in E_{0}(H)$. Now,

$$
\begin{aligned}
f^{i}-h_{0} & =U_{i}^{*}(1-Q) g \in U_{i}^{*} \sum_{n=1}^{\infty} \beta_{n}(Q)(H)=U_{i}^{*} \sum_{n=1}^{\infty} \bigoplus\left[M_{n} Q g\right] \\
& =\sum_{n=1}^{\infty} \bigoplus\left[M_{n} h_{0}\right] \subseteq \sum_{n=1}^{\infty} E_{n}(H) .
\end{aligned}
$$

Hence $h_{0}=E_{0} f^{i}$.

Let $F_{n}^{i}$ be the projection onto $\left[M_{n} h_{0}\right]$. Then $U_{i}\left[M_{n} h_{0}\right]=\left[M_{n} Q g\right]=\beta_{n}(Q)(H)$. Hence $U_{i} F_{n}^{i} U_{i}^{*}=\beta_{n}(Q)$. As the initial subspace of $U_{i}$ is $\left[M f^{i}\right]=\left[M\left[H^{\infty} f^{i}\right]\right]=$ $\left[M\left[H^{\infty} h_{0}\right]\right]=\left[M h_{0}\right]=\sum_{n=-\infty}^{\infty} F_{n}^{i}(H), U_{i} E_{n} U_{i}^{*}=U_{i} F_{n}^{i} U_{i}^{*}=\beta_{n}(Q)$. In particular $U_{1} E_{n} U_{1}^{*}=U_{2} E_{n} U_{2}^{*}$ for each $n \in \mathbf{Z}$. Consequently $U_{1} W_{t} U_{1}^{*}=U_{2} W_{t} U_{2}^{*}$ for each $t \in \mathbf{T}$ and $U_{2}^{*} U_{1} W_{t} U_{1}^{*} U_{1}=U_{2}^{*} U_{2} W_{t} U_{2}^{*} U_{1}$. But $U_{1}^{*} U_{1}, U_{2}^{*} U_{2} \in R_{0}$, hence $U_{2}^{*} U_{1} W_{t}=W_{t} U_{2}^{*} U_{1}, t \in \mathbf{T}$. Thus $U_{2}^{*} U_{1} \in R_{0}$. 
LEMMA 3.3. Suppose $g$ lies in $H^{2}$. Then there is a partial isometry $U \in H^{\infty}(\gamma)$ such that $U^{*} U \in R_{0}, U^{*} g \in H^{2}$ and $\mathcal{M}\left(U^{*} g\right)=\mathcal{M}\left(E_{0} U^{*} g\right)$.

ProOF. Since $g \in H^{2}, P(g) \in \mathcal{P}_{3}$ and we can construct a partial isometry $U \in R$ as in Proposition 3.1. It is left to show that $U$, as constructed there, lies in $H^{\infty}(\gamma)$. Let $P(g)$ be $\sum_{n=0}^{\infty} \beta_{n}(Q)$, for some $Q \in P_{1}$, then, from the way $U$ was constructed, $U E_{0} f=Q g$ where $f=U^{*} g$. Therefore $U\left[M_{n} E_{0} f\right]=\left[M_{n} Q g\right] \subseteq\left[M_{n} H^{2}\right] \subseteq$ $P_{n}(H)$. Since the initial subspace of $U$ is $\sum_{n=-\infty}^{\infty} \bigoplus\left[M_{n} E_{0} f\right], U E_{n} \bar{U}^{*} \leq P_{n}$ and, consequently, $U P_{n} U^{*} \leq P_{n}$. Hence $U \in R \cap \operatorname{Alg}\left\{P_{n}: n \in \mathbf{Z}\right\}=H^{\infty}(\gamma)$ (by Lemma 2.6).

COROLlaRY 3.4. Let $g$ be in $H^{2}$. Then there are partial isometries $U \in$ $H^{\infty}(\gamma), V \in H^{\infty}(\alpha)$, such that:

(1) $U^{*} g$ and $V^{*} g$ lie in $H^{2}$.

(2) $U U^{*} g=V V^{*} g=g$.

(3) $U^{*} U \in R_{0}, V^{*} V \in M_{0}$.

(4) $\left[H^{\infty}(\alpha) U^{*} g\right]=\left[H^{\infty}(\alpha) E_{0} U^{*} g\right]$ and $\left[H^{\infty}(\gamma) V^{*} g\right]=\left[H^{\infty}(\gamma) E_{0} V^{*} g\right]$.

PROOF. The assertions for $U$ follow from Lemma 3.3 and the assertions for $V$ follow from Lemma 3.3 applied to $\{R, \gamma\}$ in place of $\{M, \alpha\}$.

Let $M$ be the algebra $L^{\infty}(\mathbf{T})$ acting on $L^{2}(\mathbf{T})$ and let $\left\{\alpha_{t}\right\}_{t \in \mathbf{T}}$ be defined by translations. Then $H^{\infty}(\alpha)$ is the classical algebra $H^{\infty}$. In this case $M=R$ and $\alpha=\gamma$. An element $f \in H^{2}$ is called outer, in this case, if $\left[H^{\infty} f\right]=H^{2}$. But this is equivalent to $\left[H^{\infty} f\right]=\left[H^{\infty} E_{0} f\right] \neq 0$ (note that in this case, $E_{0} f$ is just the zeroth Fourier coefficient of $f$ ). Hence Lemma 3.3 is the inner-outer factorization in the classical case. It is also known, in this case, that $f \in H^{2}$ is outer if and only if $\exp \int \log |f| d t=\left|E_{0} f\right| \neq 0$. Since, by Szëgo's theorem, $\exp \int \log |f| d t=\inf \left\{\|f-g\|: g \in\left[H_{0}^{\infty} f\right]\right\}, f \in H^{2}$ is outer if and only if $\left|E_{0} f\right|=$ $\inf \left\{\|f-g\|: g \in\left[H_{0}^{\infty} f\right]\right\} \neq 0$.

In our more general setting we say that $f \in H^{2}$ is $R$-outer if $\left[H^{\infty}(\alpha) f\right]=$ $\left[H^{\infty}(\alpha) E_{0} f\right] \neq\{0\}$ and $M$-outer if $\left[H^{\infty}(\gamma) f\right]=\left[H^{\infty}(\gamma) E_{0} f\right] \neq\{0\}$. Also we say that $U \in R$ is $R$-inner if $U$ is a partial isometry in $H^{\infty}(\gamma)$ with $U^{*} U \in R_{0}$ and $V \in M$ is $M$-inner if $V$ is a partial isometry in $H^{\infty}(\alpha)$ with $V^{*} V \in M_{0}$. Then Corollary 3.4 presents the $M$-inner-outer and the $R$-inner-outer factorizations (which are the classical inner-outer factorization if $M=L^{\infty}(\mathbf{T})$ and $\alpha$ acts by translations). The next proposition is analogous to the characterization of an outer function, mentioned above, in the classical case.

Proposition 3.5. For $f \in H^{2}$ the following conditions are equivalent:

(1) $f$ is R-outer (resp. $f$ is $M$-outer).

(2) $\left[H^{\infty}(\alpha) f\right]=N_{0} H^{2}$, where $N_{0}$ is a nonzero projection in $R_{0}$ (resp. $\left[H^{\infty}(\gamma) f\right]$ $\left.=N_{0} H^{2}, 0 \neq N_{0} \in M_{0}\right)$.

(3) $\left\|E_{0} f\right\|=\inf \left\{\|(1-a) f\|: a \in H_{0}^{\infty}(\alpha)\right\} \neq 0\left(\right.$ resp. $\left\|E_{0} f\right\|=\inf \{\|(1-a) f\|: a \in$ $\left.\left.H_{0}^{\infty}(\gamma)\right\} \neq 0\right)$.

PROOF. It will suffice to prove the $R$-version.

(1) implies (2). Suppose $f \in H^{2}$ is $R$-outer. Then $\left[H^{\infty}(\alpha) f\right]=\left[H^{\infty}(\alpha) E_{0} f\right]=$ $\sum_{n=0}^{\infty} \bigoplus\left[M_{n} E_{0} f\right]$. Let $F_{0}$ be the projection onto $\left[M_{0} E_{0} f\right]$. Then $\beta_{n}\left(F_{0}\right)(H)=$ 
$\left[M_{n} E_{0} f\right]$ for all $n \in \mathbf{Z}$. Let $N_{0}$ be $\sum_{n=-\infty}^{\infty} \beta_{n}\left(F_{0}\right)$. Then

$$
\beta_{m}\left(N_{0}\right)=f_{m} \sum_{n=-\infty}^{\infty} \beta_{n+m}\left(F_{0}\right)=f_{m} N_{0} \quad \text { for each } m \in \mathbf{Z} .
$$

Hence $N_{0} \in R$. Also, as $\beta_{n}\left(F_{0}\right) \leq E_{n}$ for each $n \in \mathbf{Z}, N_{0} \in\left\{E_{n}: n<\mathbf{Z}\right\}^{\prime}$. Thus $N_{0} \in R_{0}$. Now $N_{0} H^{2}=\sum_{n=0}^{\infty} \beta_{n}\left(F_{0}\right)(H)=\left[H^{\infty}(\alpha) E_{0} f\right]=\left[H^{\infty}(\alpha) f\right]$.

(2) implies (3). Suppose $\left[H^{\infty}(\alpha) f\right]=N_{0} H^{2}$. Then

$$
\left[H_{0}^{\infty}(\alpha) f\right]=\left[H_{0}^{\infty}(\alpha)\left[H^{\infty}(\alpha) f\right]\right]=\left[H_{0}^{\infty}(\alpha) N_{0} H^{2}\right]=N_{0} H^{2} .
$$

Hence

$$
\inf \left\{\|(1-a) f\|: a \in H_{0}^{\infty}(\alpha)\right\}=\inf \left\{\|f-g\|: g \in N_{0} H^{2}\right\} .
$$

Since $f=N_{0} f$,

$$
\inf \left\{\|(1-a) f\|: a \in H_{0}^{\infty}(\alpha)\right\}=\inf \left\{\|f-g\|: g \in H_{0}^{2}\right\}=\left\|E_{0} f\right\| .
$$

If $\left\|E_{0} f\right\|=0$ then $f \in H_{0}^{2}$ and $\left[H^{\infty}(\alpha) f\right] \subseteq H_{0}^{2}$. Hence $N_{0} H^{2} \subseteq H_{0}^{2}$. But $N_{0} E_{0} \subseteq E_{0}\left(\right.$ as $\left.N_{0} \in R_{0}\right)$. Therefore

$$
N_{0}=\sum_{n=-\infty}^{\infty} N_{0} E_{n}=\sum_{n=-\infty}^{\infty} N_{0} \beta_{n}\left(E_{0}\right)=\sum_{n=-\infty}^{\infty} \beta_{n}\left(N_{0} E_{0}\right)=0 .
$$

(3) implies (1). Suppose $\left\|E_{0} f\right\|=\inf \left\{\|(1-a) f\|: a \in H_{0}^{\infty}(\alpha)\right\}$. Let $P$ be the projection onto $\left[H_{0}^{\infty}(\alpha) f\right]$. Then $P \leq P_{1}$ and $(1-P) f=E_{0} f+\left(P_{1}-P\right) f$. Hence

$$
\left(\inf \left\{\|(1-a) f\|: a \in H_{0}^{\infty}(\alpha)\right\}\right)^{2}=\|(1-P) f\|^{2}=\left\|E_{0} f\right\|^{2}+\left\|\left(P_{1}-P\right) f\right\|^{2} .
$$

Thus $P_{1} f=P f$ and $E_{0} f=(1-P) f \in\left[H^{\infty}(\alpha) f\right]$. It is left to show that $f \in\left[H^{\infty}(\alpha) E_{0} f\right]$. Let $F_{m}$ be the projection onto $\left[M_{m} E_{0} f\right]$. Then $F_{m} \leq E_{m}$ and $\sum_{m=0}^{\infty} F_{m}(H)=\left[H^{\infty}(\alpha) E_{0} f\right]$. Suppose $E_{m} f \in\left[H^{\infty}(\alpha) E_{0} f\right]$ for all $0 \leq$ $m<j$ (note that $E_{0} f \in\left[H^{\infty}(\alpha) E_{0} f\right]$ ). Then for each $a \in H_{0}^{\infty}(\alpha), E_{j} a f=$ $\sum_{m=-\infty}^{\infty} E_{j} a E_{m} f=\sum_{m=0}^{j-1} E_{j} a E_{m} f$. But $a E_{m} f \in\left[H_{0}^{\infty}(\alpha)\left[H^{\infty}(\alpha) E_{0} f\right]\right] \subseteq$ $\left[H^{\infty}(\alpha) E_{0} f\right]$. Hence

$$
E_{j} a E_{m} f \in E_{j}\left[H^{\infty}(\alpha) E_{0} f\right]=E_{j} \sum_{m=0}^{\infty} F_{m}(H)=F_{j}(H) \subseteq\left[H^{\infty}(\alpha) E_{0} f\right] .
$$

Therefore $E_{j}$ af $\in\left[H^{\infty}(\alpha) E_{0} f\right]$. This induction argument shows that $E_{j}$ af $\in$ $\left[H^{\infty}(\alpha) E_{0} f\right]$ for all $a \in H_{0}^{\infty}(\alpha), j \geq 0$. Hence $\left[H_{0}^{\infty}(\alpha) f\right] \subseteq\left[H^{\infty}(\alpha) E_{0} f\right]$. Since $f=E_{0} f+P_{1} f=E_{0} f+P f$ and $P f \in\left[H_{0}^{\infty}(\alpha) f\right]$, we are done.

COROLlaRY 3.6. Suppose $g \in H$. Then the invariant subspace $\left[H^{\infty}(\alpha) g\right]$ can be written as the orthogonal sum of a reducing subspace $\mathcal{M}_{0}$ (i.e. a $\mathcal{M}_{0} \subseteq \mathcal{M}_{0}$ for all $a \in M$ ) and a space of the form $U H^{2}$, where $U$ is a partial isometry in $R$ with $U^{*} U \in R_{0}$.

ProOF. Let $P(g)$ be the projection onto $\left[H^{\infty}(\alpha) g\right]$. Then

$$
P(g)=\sum_{n=0}^{\infty} \beta_{n}(\delta(P(g)))+F_{0}
$$


where $F_{0}$ is a projection in $R$, orthogonal to $\sum_{n=-\infty}^{\infty} \beta_{n}(\delta(P(g)))$. Hence

$$
\left[H^{\infty}(\alpha)\left(1-F_{0}\right) g\right]=\left(1-F_{0}\right)\left[H^{\infty} g\right]=\sum_{n=0}^{\infty} \beta_{n}(Q)(H)
$$

(for $\left.Q=\delta(P(g)) \in P_{1}\right)$. Hence $\left(1-F_{0}\right) g$ has a representation $\left(1-F_{0}\right) g=U f$ as described in Proposition 3.1. Thus

$$
\left[H^{\infty}(\alpha)\left(1-F_{0}\right) g\right]=U\left[H^{\infty}(\alpha) f\right]=U\left[H^{\infty}(\alpha) E_{0} f\right]=U H^{2}
$$

and

$$
\left[H^{\infty}(\alpha) g\right]=\left[H^{\infty}(\alpha) F_{0} g\right] \oplus U H^{2}=F_{0}(H) \oplus U H^{2} .
$$

We now define $a \in M$ (resp. $a \in R$ ) to be $M$-outer (resp. $R$-outer) if $a \xi$ is $M$-outer (resp. $R$-outer).

Proposition 3.7. For $a \in M$ the following conditions are equivalent.

(1) There is a partial isometry $U \in M$ such that $U^{*} a \in H^{\infty}(\alpha)$ and $U U^{*} a=a$.

(2) Let $P$ be the projection onto $\left[a H^{2}\right]$. Then $P=\sum_{n=0}^{\infty} \eta_{n}(Q)$ for some $R$ wandering projection $Q \in R_{0}^{\prime}$.

(3) $\bigcap_{n}\left[a P_{n}(H)\right]=\{0\}$.

(4) There is a partial isometry $U \in M$ such that $U^{*} a \in H^{\infty}(\alpha)$ is $M$-outer and $U U^{*} a=a$.

Moreover, if (4) is satisfied and $U_{1}, U_{2}$ are two such partial isometries such that the initial subspace of $U_{i}$ is $\left[U_{i}^{*} a H\right]$, then $U_{1}^{*} U_{2}$ and $U_{2}^{*} U_{1}$ lie in $M_{0}$.

ProOF. Note that $\left[a H^{2}\right]=\left[a H^{\infty}(\gamma) \xi\right]=\left[H^{\infty}(\gamma) a \xi\right]$ and $\left[U_{i}^{*} a H\right]=\left[U_{i}^{*} a R \xi\right]=$ $\left[R U_{i}^{*} a \xi\right]$. Hence the equivalence of (1), (2) and (4) and the uniqueness statement follow from Propositions 3.1 and 3.2 (with $\{M, \alpha\}$ replaced by $\{R, \gamma\}$ ). We now show that (2) is equivalent to (3). For this note that $P=\sum_{n=0}^{\infty} \eta_{n}(Q)$ for some $R$-wandering projection $Q \in R_{0}^{\prime}$ if and only if $\bigwedge_{m=0} \bigvee_{n \geq m} \eta_{n}(P)=0$ (Lemma 2.5). But

$$
\begin{aligned}
\bigvee_{n \geq m} \eta_{n}(P) & =\bigvee_{n \geq m}\left(\bigvee\left\{u P u^{*}: u \in R_{n} \text { is a partial isometry }\right\}\right) \\
& =\bigvee\left\{u P u^{*}: u \text { is a partial isometry in } \bigcup_{n \geq m} M_{n}\right\} .
\end{aligned}
$$

Since $R^{\gamma}[m, \infty)$ is generated by the partial isometries in $\bigcup_{n \geq m} M_{n}$ (as a $\sigma$-weakly closed linear space), $\bigvee_{n \geq m} \eta_{n}(P)$ is the projection onto

$$
\left[R^{\gamma}[m, \infty) P(H)\right]=\left[R^{\gamma}[m, \infty)\left[H^{\infty}(\gamma) a \xi\right]\right]=\left[R^{\gamma}[m, \infty) a \xi\right]=\left[a P_{m}(H)\right]
$$

for $m \geq 0$. Hence $\bigwedge_{m=0} \bigvee_{n \geq m} \eta_{n}(P)=0$ if and only if $\bigcap_{m>n}\left[a P_{m}(H)\right]=\{0\}$. Since $\bigcap_{m \in \mathbf{Z}}\left[a P_{m}(H)\right]=\{0\}$ if and only if $\bigcap_{m>0}\left[a P_{m}(H)\right]=\{0\}$, we are done.

The previous proposition presents the factorization for operators $a \in M$ and its uniqueness. Consider now the nest algebra $A=\operatorname{Alg}\left\{P_{n}: n \in \mathbf{Z}\right\}$ in $B(H)$. In [3, Theorem 3.3] Arveson presented a factorization for operators $A$ in $B(H)$ (that satisfy a certain condition) as $A=U B$, where $U$ is a partial isometry in $B(H)$ with $U^{*} U \in A \cap A^{*}$ and $B$ is outer (in the sense that $\left[A P_{n}(H)\right]=[A H] \cap P_{n}(H)$ and the projection onto $[A H]$ lies in $\left.A \cap A^{*}\right)$. The condition for $A \in B(H)$ to have such a factorization is $\bigcap_{n}\left[A P_{n}(H)\right]=\{0\}$. Hence Proposition 3.7 shows that $a \in M$ 
satisfies our condition for factorization if and only if it satisfies Arveson's condition for factorization (with respect to $A=\operatorname{Alg}\left\{P_{n}: n \in \mathbf{Z}\right\}$ ). The next result shows that $b \in H^{\infty}(\alpha)$ is $M$-outer if and only if it is outer in Arveson's terminology.

Proposition 3.8. For $b \in H^{\infty}(\alpha)$ the following statements are equivalent:

(1) $b$ is $M$-outer.

(2) $\left[b H^{2}\right]=\left[\varepsilon_{0}(b) H^{2}\right] \neq\{0\}$.

(3) $\left[b P_{n}(H)\right]=\left[\varepsilon_{0}(b) P_{n}(H)\right]$ for each $n \in \mathbf{Z}$ and $\left[\varepsilon_{0}(b) H^{2}\right] \neq\{0\}$.

(4) $\left[b H^{2}\right]=Q H^{2}$ for some nonzero projection $Q \in M_{0}$.

(5) $0 \neq\left\|\varepsilon_{0}(b) \xi\right\|=\inf \left\{\|b \xi-g\|: g \in\left[b H_{0}^{2}\right]\right\}$.

(6) The projection onto $[b H]$ lies in $M_{0}$ and, for each $n \in \mathbf{Z},\left[b P_{n}(H)\right]=[b H] \cap$ $P_{n}(H)$.

PROOF. The equivalence of (1), (2), (4) and (5) follows from Proposition 3.5 with the observations that

$$
\begin{gathered}
{\left[b H^{2}\right]=\left[b H^{\infty}(\gamma) \xi\right]=\left[H^{\infty}(\gamma) b \xi\right]} \\
{\left[\varepsilon_{0}(b) H^{2}\right]=\left[\varepsilon_{0}(b) H^{\infty}(\gamma) \xi\right]=\left[H^{\infty}(\gamma) \varepsilon_{0}(b) \xi\right]=\left[H^{\infty}(\gamma) E_{0} b \xi\right]}
\end{gathered}
$$

and

(2) implies (3).

$$
\begin{aligned}
{\left[p P_{n}(H)\right] } & =\left[b R^{\gamma}[n, \infty) \xi\right]=\left[R^{\gamma}[n, \infty) b \xi\right]=\left[R^{\gamma}[n, \infty)\left[H^{\infty}(\gamma) b \xi\right]\right] \\
& =\left[R^{\gamma}[n, \infty)\left[H^{\infty}(\gamma) \varepsilon_{0}(b) \xi\right]\right]=\left[R^{\gamma}[n, \infty) \varepsilon_{0}(b) \xi\right]=\left[\varepsilon_{0}(b) P_{n}(H)\right] .
\end{aligned}
$$

(3) implies (2) is trivial.

(3) implies (6). $[b H]=\bigvee\left\{\left[b P_{n}(H)\right]: n \in \mathbf{Z}\right\}=\bigvee\left\{\left[\varepsilon_{0}(b) P_{n}(H)\right]: n \in \mathbf{Z}\right\}=$ $\left[\varepsilon_{0}(b) H\right]$. Hence the projection onto $[b H]$ lies in $M_{0}$. Also $[b H] \cap P_{n}(H)=P_{n}[b H]=$ $P_{n}\left[\varepsilon_{0}(b) H\right]=\left[\varepsilon_{0}(b) P_{n}(H)\right]=\left[b P_{n}(H)\right]$.

(6) implies (4). Let $Q \in M_{0}$ be the projection onto $[b H]$. Then $\left[b H^{2}\right]=[b H] \cap$ $P_{0}(H)=Q(H) \cap P_{0}(H)=Q P_{0}(H)=Q H^{2}$.

LEMMA 3.9. Suppose $a \in H^{\infty}(\alpha)$ is invertible; then $a$ is $M$-outer if and only if $a^{-1} \in H^{\infty}(\alpha)$.

Proof. Suppose $a^{-1} \in H^{\infty}(\alpha)$; then $\left[a H^{2}\right]=H^{2}$ and, hence, $a$ is outer by Proposition 3.8(4). Suppose now that $a$ is invertible and $M$-outer. Then $H=[a H]=\left[\varepsilon_{0}(a) H\right]$. Hence $\xi \in\left[\varepsilon_{0}(a) H\right]$ and, as $P_{0} \xi=\xi, \xi \in P_{0}\left[\varepsilon_{0}(a) H\right]=$ $\left[\varepsilon_{0}(a) H^{2}\right]=\left[a H^{2}\right]$. Hence there are $\left\{x_{n}\right\} \subseteq H^{\infty}(\alpha)$ such that $a x_{n} \xi \rightarrow \xi$ and, by applying $a^{-1}, x_{n} \xi \rightarrow a^{-1} \xi$. But $x_{n} \xi \in H^{2}$, hence $a^{-1} \xi \in H^{2}$. For $n<0$, $\varepsilon_{n}\left(a^{-1}\right) \xi=E_{n} a^{-1} \xi=0$. Thus $a^{-1} \in H^{\infty}(\alpha)$.

The following result was proved in [3] for nest algebras and in [1] for maximal finite subdiagonal algebras.

THEOREM 3.10. Suppose $a \in M$ is invertible. Then we can write $a=u b$, where $u$ is unitary in $M$ and $b \in H^{\infty}(\alpha) \cap H^{\infty}(\alpha)^{-1}$.

PROOF. First note that if $x \in \bigcap_{n}\left[a P_{n}(H)\right]=\bigcap_{n}\left\{a y: y \in P_{n}(H)\right\}$, then $a^{-1} x \in$ $\bigcap_{n} P_{n}(H)=\{0\}$. Hence $\bigcap_{n}\left[a P_{n}(H)\right]=\{0\}$ and we can apply Proposition 3.7 to write $a=w c$, where $w$ is a partial isometry in $M, w^{*} w c=c$ and $c \in H^{\infty}(\alpha)$ is $M$ outer. Now let $\varepsilon_{0}(c)=v\left|\varepsilon_{0}(c)\right|$ be the polar decomposition of $\varepsilon_{0}(c)$, let $b \in H^{\infty}(\alpha)$ 
be $v^{*} c$, and let $u$ be $w v$. The final projection of $v$ is the projection onto $\left[\varepsilon_{0}(c) H\right]$. The initial subspace of $w$ is $[c H]$ and, since $c$ is $M$-outer, $[c H]=\left[\varepsilon_{0}(c) H\right]$. Therefore $u=w v$ is a partial isometry in $M$ with initial subspace $\left[\left|\varepsilon_{0}(c)\right| H\right]$ and final subspace $[a H]$. We have $a=w c=b u$ and $\left[b H^{2}\right]=v^{*}\left[c H^{2}\right]=v^{*}\left[\varepsilon_{0}(c) H^{2}\right]=\left[\varepsilon_{0}(b) H^{2}\right]$. Hence $b$ is outer and has the further propety that $\varepsilon_{0}(b) \geq 0$.

Now note that, since $a=u b$ is invertible, $b$ is bounded below. Hence Ker $b$ $(=\{x \in H: b x=0\})=\{0\}$ and, for each closed linear subspace $K \subseteq H,\{b x: x \in$ $K\}$ is closed. If $\varepsilon_{0}(b) x=0$ for some $x \in E_{0}(H)$, then $0 \neq b x \in H_{0}^{2} \cap\left[b H^{2}\right]=\left[b H_{0}^{2}\right]$ (as $b$ is $M$-outer) and, since $\left[b H_{0}^{2}\right]=\left\{b x: x \in H_{0}^{2}\right\}$, there is some $y \in H_{0}^{2}$ such that $b x=b y$. Since Ker $b=\{0\}, x=y=0$. Hence Ker $\varepsilon_{0}(b)=\{0\}$.

Let $y$ be in $\left[\varepsilon_{0}(b) E_{0}(H)\right] \subseteq\left[\varepsilon_{0}(b) H^{2}\right]=\left[b H^{2}\right]=\left\{b z: z \in H^{2}\right\}$. Then $y=b z$ for some $z \in H^{2}$. But $y \in E_{0}(H)$, hence $y=\varepsilon_{0}(b) E_{0} z \in\left\{\varepsilon_{0}(b) z: z \in E_{0}(H)\right\}$. Consequently the range of $\varepsilon_{0}(b)$, as an operator on $E_{0}(H)$, is closed. We know also that $\operatorname{Ker} \varepsilon_{0}(b)=\{0\}$ and that $\varepsilon_{0}(b) \geq 0$. Therefore $\varepsilon_{0}(b)$ is invertible. Thus $H=\left[\varepsilon_{0}(b) H\right]=[b H]=\{b x: x \in H\}$. Hence $b$ is invertible. By the previous lemma $b \in H^{\infty}(\alpha) \cap H^{\infty}(\alpha)^{-1}$.

COROLLARY 3.11. Every invertible positive operator $a \in M$ can be factored in the form $a=b^{*} b$, where $b \in H^{\infty}(\alpha) \cap H^{\infty}(\alpha)^{-1}$.

PROOF. Let $a^{1 / 2}$ be the positive square root of $a$. Then $a^{1 / 2}$ is invertible and by the last theorem we can write $a^{1 / 2}=u b$ for some partial isometry $u \in M$ such that $u^{*} u b=b$ and $b \in H^{\infty}(\alpha) \cap H^{\infty}(\alpha)^{-1}$. Hence $a=a^{1 / 2} a^{1 / 2}=b^{*} u^{*} u \dot{b}=b^{*} b$.

Note that, although most of our analysis depends on the special representation of $M$ that was chosen (with a cyclic and separating vector $\xi$ and such that $\{M, \alpha\}$ is a canonical pair), Theorem 3.10 and Corollary 3.11 do not depend on the representation and hold in general.

4. An expectation and its application. In this section we construct an expectation from the von Neumann algebra $R_{0}^{\prime}$ (that contains $M$ ) onto $M$ that maps the nest subalgebra $R_{0}^{\prime} \cap \operatorname{Alg}\left\{P_{n}: n \in \mathbf{Z}\right\}$ onto $H^{\infty}(\alpha)$. We will apply it to prove two results (a distance estimate and an interpolation result).

Before constructing the expectation we want to replace $\alpha$ by another flow, $\tilde{\alpha}$, with a special property. For this we will need the following discussion which will be summarized in Lemma 4.5 .

For $n \in \mathbf{Z}$ let $c_{n} \in Z\left(M_{0}\right)$ be defined as follows

$$
c_{n}= \begin{cases}f_{n} \sum_{m=0}^{n-1} f_{m}, & n>0, \\ 0, & n=0, \\ -f_{n} \sum_{m=n}^{-1} f_{m}, & n<0 .\end{cases}
$$

Let $\tilde{W}_{t}, t \in T$, be the unitary operator $\sum_{n=-\infty}^{\infty} \exp \left(i t c_{n}\right) E_{n}$. Then, it was shown in $\left[\mathbf{1 5}\right.$, Lemma 3.8] that $\left\{\tilde{W}_{t}\right\}_{t \in T}$ implements a flow $\tilde{\alpha}$ on $M$ with $\tilde{\alpha}_{t}(a)=\exp \left(i t c_{n}\right) a$ for $a \in M_{n}$.

Let $c_{n}=\sum_{m=-\infty}^{\infty} m h_{m, n}$ be the spectral decomposition of $c_{n}, n \in \mathbf{Z}$. Then $h_{m, n} \neq 0$ only if $0 \leq m \leq n$. Also note that $h_{0, n}=1-f_{n}$. For $a \in M_{n}, a=f_{n} a$ and one easily gets from this that $\operatorname{sp}_{\tilde{\alpha}}(a) \subseteq\{1,2, \ldots, n\}$ (if $n>0$ ), $\operatorname{sp}_{\tilde{\alpha}}(a)=0$ (if $n=0$ ) and $\operatorname{sp}_{\tilde{\alpha}}(a) \subseteq\{n, n+1, \ldots,-1\}$ (if $n<0$ ). The following lemma follows immediately. 
LeMmA 4.1. $H^{\infty}(\alpha)=H^{\infty}(\tilde{\alpha}), H_{0}^{\infty}(\alpha)=H_{0}^{\infty}(\tilde{\alpha})$ and $M^{\tilde{\alpha}}(\{0\})=M_{0}$.

We have

$$
\tilde{W}_{t}=\sum_{n} \exp \left(i t c_{n}\right) E_{n}=\sum_{n} \sum_{m} \exp (i t m) h_{m, n} E_{n}=\sum_{m} e^{i t m} \sum_{n} h_{m, n} E_{n}
$$

Hence, if we write $\tilde{E}_{n}$ for the projection onto $M^{\tilde{\alpha}}(\{n\})$ (to be denoted by $\tilde{M}_{n}$ ), then $\left\{\tilde{E}_{n}\right\}$ are the spectral projections of $\tilde{W}_{t}$ and we have

$$
\tilde{E}_{m}=\sum_{n} h_{m, n} E_{n} .
$$

(In fact $\tilde{E}_{m}=\sum_{n=m}^{\infty} h_{m, n} E_{n}$ if $m>0, \tilde{E}_{m}=\sum_{n=-\infty}^{\infty} h_{m, n} E_{n}$ if $m<0$ and $\tilde{E}_{0}=E_{0}$.)

LeMmA 4.2. For $j, m, n \in \mathbf{Z}$ with $m \neq n, j \neq 0$, we have $h_{j, m} h_{j n}=0$.

ProOF. We will assume that $j>0$. The proof for $j<0$ is similar. As $j>0$ we can suppose that $m>n>0$. Let $q$ be $h_{j, m} h_{j, n}$. Then $c_{m} q=c_{n} q=j q$ and $q \leq f_{n} f_{m}$. But then

$$
\begin{aligned}
j q & =\left(\sum_{i=0}^{m-1} f_{i}\right) q=\left(\sum_{i=0}^{n-1} f_{i}\right) q+\left(\sum_{i=n}^{m-1} f_{i}\right) q \\
& \geq\left(\sum_{i=0}^{n-1} f_{i}\right) q+f_{n} q=\left(\sum_{i=0}^{n-1} f_{i}\right) q+q
\end{aligned}
$$

and $\left(\sum_{i=0}^{n-1} f_{i}\right) q=j q$. This leads to a contradiction if $q \neq 0$.

LEMMA 4.3 . (1) $\sum_{j=1}^{n} h_{m, j} \geq \sum_{j=1}^{n} h_{m+1, j}$ for $n>0$ and $m>0$.

(2) $\sum_{j=n}^{-1} h_{m-1, j} \leq \sum_{j=n}^{-1} h_{m, j}$ for $n<0$ and $m<0$.

PROOF. We will prove assertion (1). The proof of (2) is similar and will be omitted. Fix $n>0, m>0$ and $n \geq j \geq 1$. Then $c_{j} h_{m+1, j}=(m+1) h_{m+1, j}$. As $c_{j}=f_{j} \sum_{k=0}^{j-1} f_{k}$, we can write $h_{m+1, j}$ as a sum of orthogonal projections $\left\{p_{s}\right\}_{s=2}^{j}$ in $Z\left(M_{0}\right)$ such that $f_{j}\left(\sum_{k=0}^{s-1} f_{k}\right) p_{s}=(m+1) p_{s}$ and $f_{j}\left(\sum_{k=0}^{s-2} f_{k}\right) p_{s}=m p_{s}$. Hence $f_{j} f_{s-1} p_{s}=p_{s}$ and $c_{s-1} p_{s}=f_{s-1}\left(\sum_{k=0}^{s-2} f_{k}\right) p_{s}=f_{j}\left(\sum_{k=0}^{s-2} f_{k}\right) p_{s}=m p_{2}$. Thus $p_{s} \leq h_{m, s-1} \leq \sum_{i=1}^{n} h_{m, i}$. As $h_{m+1, j}=\sum_{s=2}^{j} p_{s}, h_{m+1, j} \leq \sum_{i=1}^{n} h_{m, i}$. Since this holds for each $1 \leq j \leq n$ we are done.

Now recall that $\tilde{E}_{m}=\sum_{n} h_{m, n} E_{n}$ and let $\tilde{f}_{n}$ be the projections (in $Z\left(\tilde{M}_{0}\right)=$ $\left.Z\left(M_{0}\right)\right)$ defined by $\tilde{f}_{n}=\sup \left\{u u^{*}: u\right.$ is a partial isometry in $\left.\tilde{M}_{n}\right\}, n \in \mathbf{Z}$. By Lemma $2.7, \tilde{f}_{n}=c\left(\tilde{E}_{n}\right)$ and, thus, $\tilde{f}_{n}=\sum_{j} h_{n, j} f_{j}$.

For $n>0, \tilde{f}_{n}=\sum_{j=1}^{\infty} h_{n, j}\left(\right.$ as $h_{n, j}=h_{n, j} f_{j}$ for $\left.n \neq 0\right)$ and for $n<0$, $\tilde{f}_{n}=\sum_{j=-\infty}^{-1} h_{n, j}$. Also $\tilde{f}_{0}=I$. Using Lemma 4.3 we immediately have

LEMMA 4.4. For $n \geq 0, \tilde{f}_{n+1} \leq \tilde{f}_{n}$ and for $n<0, \tilde{f}_{n} \leq \tilde{f}_{n+1}$.

When a canonical pair $\{B, \eta\}$ satisfies the property of Lemma 4.4 (for the corresponding projections) we say that it satisfies the roof condition. 
Now let $\tilde{P}_{n}$ be $\sum_{j=n}^{\infty} \tilde{E}_{j}$ for $n \in \mathbf{Z}$ (or, equivalently, $\tilde{P}_{n}$ is the orthogonal projection onto $\left.\left[M^{\tilde{\alpha}}[n, \infty) \xi\right]\right)$. Then, for $n \in \mathbf{Z}$,

$$
\tilde{P}_{n}=\sum_{j=n}^{\infty} \tilde{E}_{j}=\sum_{j=n}^{\infty} \sum_{i} h_{j, i} E_{i}=\sum_{i}\left(\sum_{j=n}^{\infty} h_{j, k}\right) E_{i}
$$

Fix $n>0$ and let $r_{i}=\sum_{j=n}^{\infty} h_{j, i}$ and $t_{i}=r_{i}+\bigvee_{j=n}^{i-1} r_{j}\left(1-f_{i}\right)$ for $i \geq n$ (where $\left.t_{n}=r_{n}\right)$. Then, as $E_{i} \leq f_{i}$ for each $i \in \mathbf{Z}$,

$$
\tilde{P}_{n}=\sum t_{i} E_{i}
$$

From the definition of $\left\{c_{m}\right\}$, we have $r_{i} \leq f_{i}$ and $f_{i} f_{i+k} r_{i} \leq r_{i+k}$, for $i>0$ and $k>0$. Hence, for $i>n$ and $k>0, r_{i} \leq r_{i+k}+\left(1-f_{i+k}\right) r_{i}$. It follows that $t_{i} \leq t_{i+1}$ for $i \geq n$. Hence for $n>0$,

$$
\tilde{P}_{n}=t_{n} P_{n}+\left(t_{n+1}-t_{n}\right) P_{n+1}+\cdots .
$$

For $n<0$,

$$
\begin{aligned}
\tilde{P}_{n} & =\sum_{i}\left(\sum_{j=n}^{\infty} h_{j, i}\right) E_{i}=\sum_{i=-\infty}^{n-1}\left(\sum_{j=n}^{\infty} h_{j, i}\right) E_{i}+P_{n} \\
& =\sum_{i=-\infty}^{n-1}\left(\sum_{j=n}^{-1} h_{j, i}\right) E_{i}+P_{n} .
\end{aligned}
$$

Let $r_{i}^{\prime}=\sum_{j=n}^{-1} h_{j, i}$. Then we have $r_{i-k}^{\prime} \leq f_{i-k}$ and $f_{i} f_{i-k} r_{i-k}^{\prime} \leq r_{i}^{\prime}$ for $i<0, k>0$. Hence $f_{i} r_{i-k}^{\prime} \leq r_{i}^{\prime}$ and $r_{i-k}^{\prime} \leq r_{i}^{\prime}+\left(1-f_{i}\right) r_{i-k}^{\prime}$. Let $t_{i}^{\prime}$ be $r_{i}^{\prime}+\left(1-f_{i}\right)\left(\bigvee_{j=-\infty}^{i-1} r_{j}^{\prime}\right)$ and, then, $t_{i}^{\prime} \leq t_{i+1}^{\prime}$ for $i \leq-2$ because $r_{i}^{\prime} \leq r_{i+1}^{\prime}+\left(1-f_{i+1}\right) r_{i}^{\prime} \leq t_{i+1}^{\prime}$ and, for $j<i$,

$$
\left(1-f_{i}\right) r_{j}^{\prime} \leq\left(1-f_{i}\right) r_{i+1}^{\prime}+\left(1-f_{i}\right)\left(1-f_{i+1}\right) r_{j}^{\prime} \leq t_{i+1}^{\prime}
$$

As $r_{i}^{\prime} E_{i}=t_{i}^{\prime} E_{i}$ we have,

(**) $\tilde{P}_{n}=\sum_{i=-\infty}^{n-1} t_{i}^{\prime} E_{i}+P_{n}=\left(1-t_{-1}^{\prime}\right) P_{n}+\left(t_{-1}^{\prime}-t_{-2}^{\prime}\right) P_{n-1}+\left(t_{-2}^{\prime}-t_{-3}^{\prime}\right) P_{n-2}+\cdots$

We wish now to get expressions similar to $\left({ }^{*}\right)$ and $\left({ }^{* *}\right)$ with the roles of $\left\{P_{n}\right\}$ and $\left\{\tilde{P}_{n}\right\}$ interchanged. Since $\tilde{E}_{m}=\sum_{m} h_{m, n} E_{n}$ and $h_{m, j} h_{m, k}=0$ for $j \neq k$, we have $\tilde{E}_{m} E_{n}=h_{m, n} E_{n}=h_{m, n} \tilde{E}_{m}$. Hence $E_{n}=\sum_{m} h_{m, n} \tilde{E}_{m}$ and $P_{n}=$ $\sum_{j=n}^{\infty}\left(\sum_{n} h_{m, j} \tilde{E}_{n}\right)=\sum_{m}\left(\sum_{j=n}^{\infty} h_{m, j}\right) \tilde{E}_{m}$. Now fix $n>0$. Then

$$
P_{n}=\sum_{m=1}^{\infty}\left(\sum_{j=n}^{\infty} h_{m, j}\right) \tilde{E}_{m}
$$

Let $q_{m}$, for $m>0$, be $1-\sum_{j=1}^{\infty} h_{m, j}$. Then $q_{m} \tilde{E}_{m}=0$ because $\tilde{E}_{m}=$ $\sum_{n=1}^{\infty} h_{m, n} E_{n}$ and

$$
q_{m}+\sum_{j=n}^{\infty} h_{m, j}=1-\sum_{i=1}^{n-1} h_{m, j} \leq 1-\sum_{j=1}^{n-1} h_{m+1, j}=q_{m+1}+\sum_{j=n}^{\infty} h_{m, j},
$$


where the inequality follows from Lemma 4.3. Let $s_{m}$ be $q_{m}+\sum_{j=n}^{\infty} h_{m, j}, m>0$. Then

$$
P_{n}=\sum_{m=1}^{\infty} s_{m} \tilde{E}_{m}=s_{1} \tilde{P}_{1}+\left(s_{2}-s_{1}\right) \tilde{P}_{2}+\left(s_{3}-s_{2}\right) \tilde{P}_{3}+\cdots
$$

for $n>0$.

Now fix $n<0$. We have

$$
P_{n}=\tilde{P}_{0}+\sum_{m=n}^{-1}\left(\sum_{j=n}^{\infty} h_{m, j}\right) \tilde{E}_{m}=\tilde{P}_{0}+\sum_{m=n}^{-1}\left(\sum_{j=n}^{-1} h_{m, j}\right) \tilde{E}_{m} .
$$

Let $s_{m}^{\prime}$ be $\sum_{j=n}^{-1} h_{m, j}$ for $m<0$. Then we have, by Lemma 4.3 , that $s_{m-1}^{\prime} \leq s_{m}^{\prime}$. Hence

$$
P_{n}=\left(1-s_{-1}^{\prime}\right) \tilde{P}_{0}+\left(s_{-1}^{\prime}-s_{-2}^{\prime}\right) \tilde{P}_{-1}+\left(s_{-2}^{\prime}-s_{-3}^{\prime}\right) \tilde{P}_{-2}+\cdots+s_{n}^{\prime} \tilde{P}_{n}
$$

for $n<0$.

We summarize all this in the following

LEMMA 4.5. There is a canonical pair $\{M, \tilde{\alpha}\}$ with the following properties:

(1) $H^{\infty}(\alpha)=H^{\infty}(\tilde{\alpha})$ and $M^{\tilde{\alpha}}(\{0\})=M_{0}\left(=M^{\alpha}(\{0\})\right)$.

(2) $\tilde{\alpha}$ satisfies the roof condition (see Lemma 4.4).

(3) For $n \in \mathbf{Z}, \tilde{P}_{n}=\sum_{m} t_{m, n} P_{n}$, where $\left\{t_{m, n}\right\}_{m=-\infty}^{\infty}$ is an orthogonal family of projections in $Z\left(M_{0}\right)$. For $n \geq 0, \tilde{P}_{n} \leq P_{n}$.

(4) For $n \in \mathbf{Z}, P_{n}=\sum s_{m, n} \tilde{P}_{n}$, where $\left\{s_{m, n}\right\}_{m=-\infty}^{\infty}$ is an orthogonal family of projections in $Z\left(M_{0}\right)$. For $n \leq 0, P_{n} \leq \tilde{P}_{n}$.

Consequently,

(5) $R \cap \operatorname{Alg}\left\{P_{n}: n \in \mathbf{Z}\right\}=R \cap \operatorname{Alg}\left\{\tilde{P}_{n}: n \in \mathbf{Z}\right\}$.

(6) $M_{0}^{\prime} \cap \operatorname{Alg}\left\{P_{n}: n \in \mathbf{Z}\right\}=M_{0}^{\prime} \cap \operatorname{Alg}\left\{\tilde{P}_{n}: n \in \mathbf{Z}\right\}$.

(Here $\tilde{P}_{n}$ is the projection onto $\left.\left[M^{\tilde{\alpha}}[n, \infty) \xi\right]\right)$.

Let $B$ be a von Neumann algebra and let $C$ be a von Neumann subalgebra of $B$. An expectation of $B$ onto $C$ is a positible linear map $\psi$ from $B$ onto $C$ such that $\psi(I)=I$ and $\psi(b c)=\psi(b) c$ for every $b \in B, c \in C$.

If $\psi: B \rightarrow C$ is an expectation onto $C$, then $\psi$ is bounded (in fact $\|\psi\|=1$ ), $\psi \circ \psi=\psi, C$ is the set of fixed points of $\psi$ and $\psi(b)^{*} \psi(b) \leq \psi\left(b^{*} b\right)$ for $b \in B$ (see [1, Appendix] for details).

THEOREM 4.6. There exists an expectation $\psi: M_{0}^{\prime} \rightarrow R\left(\right.$ resp. $\left.\psi^{\prime}: R_{0}^{\prime} \rightarrow M\right)$ such that

$$
\psi\left(M_{0}^{\prime} \cap \operatorname{Alg}\left\{P_{n}^{\prime}: n \in \mathbf{Z}\right\}\right)=R \cap \operatorname{Alg}\left\{P_{n}: n \in \mathbf{Z}\right\} \quad\left(=H^{\infty}(\gamma)\right)
$$

(resp. $\left.\psi^{\prime}\left(R_{0}^{\prime} \cap \operatorname{Alg}\left\{P_{n}: n \in \mathbf{Z}\right\}\right)=M \cap \operatorname{Alg}\left\{P_{n}: n \in \mathbf{Z}\right\}=H^{\infty}(\alpha)\right)$.

PROOF. It will suffice to prove the existence of $\psi$. Using Lemma 4.5 (and its notations) we have $M^{\tilde{\alpha}}(\{0\})=M_{0}, M_{0}^{\prime} \cap \operatorname{Alg}\left\{P_{n}: n \in \mathbf{Z}\right\}=M_{0}^{\prime} \cap \operatorname{Alg}\left\{\tilde{P}_{n}: n \in \mathbf{Z}\right\}$ and $R \cap \operatorname{Alg}\left\{P_{n}: n \in \mathbf{Z}\right\}=R \cap \operatorname{Alg}\left\{\tilde{P}_{n}: n \in \mathbf{Z}\right\}$. Hence we can prove the theorem for $\tilde{\alpha}$ in place of $\alpha$ or, equivalently, assume that $\alpha$ satisfies the roof condition (i.e., $f_{n+1} \leq f_{n}$ for $n \geq 0$ and $f_{n-1} \leq f_{n}$ for $\left.n<0\right)$. In the rest of the proof we will assume that $\alpha$ satisfies the roof condition and we write $f_{+}$for $\bigwedge\left\{f_{n}: n>0\right\}, f_{-}$ for $\bigwedge\left\{f_{n}: n<0\right\}$ and $f_{\infty}$ for $f_{+} \vee f_{-}$. 
Since $\mathbf{Z}$ is an amenable group we can assign to each $g \in l^{\infty}(\mathbf{Z})$ a number $m(g) \in \mathbf{C}$ such that $g \rightarrow m(g)$ is a linear functional, $\inf \{g(n): n \in \mathbf{Z}\} \leq m(g) \leq$ $\operatorname{Sup}\{g(n): n \in \mathbf{Z}\}$ for each real valued $g \in l^{\infty}(\mathbf{Z})$ and $m\left(g_{k}\right)=m(g)$, where $g_{k}(s)=g(k+s), k, s \in \mathbf{Z}$. In fact, we can choose a state $\rho$ on the algebra $l^{\infty}\left(\mathbf{Z}_{+}\right) / c_{0}$ and let $m(g)$ be the value of $\rho$ on the coset, in $l^{\infty}\left(\mathbf{Z}_{+}\right) / c_{0}$, of $g^{\prime} \in l^{\infty}\left(\mathbf{Z}_{+}\right)$, where $g^{\prime}(n)=(1 /(2 n+1)) \sum_{i=-n}^{n} g(i), n \geq 0$. If for some real valued $g \in l^{\infty}(\mathbf{Z})$, $g^{\prime}(n) \geq a(n)$ for $n \geq 0$ and $a(n) \rightarrow a_{0} \in \mathbf{R}$ as $n \rightarrow \infty$, then $m(g)=\rho\left(g^{\prime}+c_{0}\right) \geq$ $\rho\left(a+c_{0}\right)=\rho\left(a_{0}+c_{0}\right)=a_{0}$.

Since $\left|g^{\prime}(n)\right| \leq_{i} \operatorname{Sup}|g(i)|, n \geq 0$, we see that

$$
|m(g)|=\left|\rho\left(g^{\prime}+c_{0}\right)\right| \leq\left\|g^{\prime}+c_{0}\right\| \leq\|g\|_{\infty} .
$$

Now let $T$ be an operator in $M_{0}^{\prime}$ and $x, y$ vectors in $H$. Then $g_{x, y}(n)=$ $\left\langle\beta_{n}(T) x, y\right\rangle$ defines a function $g_{x, y} \in l^{\infty}(\mathbf{Z})$ (as $\left.\left\|\beta_{n}(T)\right\| \leq\|T\|\right)$. For each $x, y \in H$ let $[x, y]$ be $m\left(g_{x, y}\right)$. Then

$$
|[x, y]|=\left|m\left(g_{x, y}\right)\right| \leq\left\|g_{x, y}\right\|_{\infty} \leq\|T\|\|x\|\|y\| .
$$

Hence there is an operator $\psi_{0}(T)$ on $H$ such that $\left\langle\psi_{0}(T) x, y\right\rangle=m\left(g_{x, y}\right)$ for $x, y \in$ $H$, and $\left\|\psi_{0}(T)\right\| \leq\|T\|$.

For a unitary operator $u \in M_{0},\left\langle u^{*} \psi_{0}(T) u x, y\right\rangle=m\left(g_{u x, u y}\right)=m\left(g_{x, y}\right)=$ $\left\langle\psi_{0}(T) x, y\right\rangle$ as $g_{u x, u y}=\left\langle\beta_{n}(T) u x, u y\right\rangle=\left\langle u \beta_{n}(T) x, u y\right\rangle=g_{x, y}$, for all $x, y \in H$. Hence $\psi_{0}(T) \in M_{0}^{\prime}$.

To find $\beta_{m}\left(\psi_{0}(T)\right)$ for $m \in \mathbf{Z}$, consider the operator $v_{m, k} v_{m, k}^{*} \beta_{m}\left(\psi_{0}(T)\right)=$ $v_{m, k} \psi_{0}(T) v_{m, k}^{*}$ for $k \geq 1$. Then

$$
\left\langle v_{m, k} \psi_{0}(T) v_{m, k}^{*} x, y\right\rangle=\left\langle\psi_{0}(T) v_{m, k}^{*} x, v_{m, k}^{*} y\right\rangle=m(g)
$$

where

$$
\begin{aligned}
g(n) & =\left\langle\beta_{n}(T) v_{m, k}^{*} x, v_{m, k}^{*} y\right\rangle=\left\langle v_{m, k} \beta_{n}(T) v_{m, k}^{*} x, y\right\rangle=\left\langle v_{m, k} v_{m, k}^{*} \beta_{m}\left(\beta_{n}(T)\right) x, y\right\rangle \\
& =\left\langle f_{m} \beta_{m+n}(T) x, v_{m, k} v_{m, k}^{*} y\right\rangle=\left\langle\beta_{m+n}(T) x, v_{m, k} v_{m, k}^{*} y\right\rangle .
\end{aligned}
$$

Hence, as $m(g)$ is translation-invariant,

$$
\left\langle v_{m, k} \psi_{0}(T) v_{m, k}^{*} x, y\right\rangle=\left\langle\psi_{0}(T) x, v_{m, k} v_{m, k}^{*} y\right\rangle=\left\langle v_{m, k} v_{m, k}^{*} \psi_{0}(T) x, y\right\rangle .
$$

Since this holds for all $x, y \in H, v_{m, k} v_{m, k}^{*} \beta_{m}\left(\psi_{0}(T)\right)=v_{m, k} v_{m, k}^{*} \psi_{0}(T)$ for each $k \geq 1$. Hence $\beta_{m}\left(\psi_{0}(T)\right)=f_{m} \beta_{m}\left(\psi_{0}(T)\right)=f_{m} \psi_{0}(T)$. By Lemma 2.4(4), $\psi_{0}(T) \in$ $R$.

Since each $\beta_{n}$ is positive and linear and so is $m, \psi_{0}$ is a positive linear map from $M_{0}^{\prime}$ into $R$.

Suppose $T \in M_{0}^{\prime} \cap \operatorname{Alg}\left\{P_{n}: n \in \mathbf{Z}\right\}$. Then, for $n, m \in \mathbf{Z}$,

$$
\begin{aligned}
\beta_{n}(T) P_{m} & =\beta_{n}(T) f_{n} P_{m}=\beta_{n}(T) \beta_{n}\left(P_{m-n}\right)=\beta_{n}\left(T P_{m-n}\right)=\beta_{n}\left(P_{m-n} T P_{m-n}\right) \\
& =\beta_{n}\left(P_{m-n}\right) \beta_{n}(T) \beta_{n}\left(P_{m-n}\right)=P_{m} \beta_{n}(T) P_{m} .
\end{aligned}
$$

Consequently, for $x, y \in H$ and $m \in \mathbf{Z},\left\langle\psi_{0}(T) P_{m} x, y\right\rangle=m(g)$, where $g(n)=$ $\left\langle\beta_{n}(T) P_{m} x, y\right\rangle=\left\langle\beta_{n}(T) P_{m} X, P_{m} y\right\rangle$. Hence $\left\langle\psi_{0}(T) P_{m} x, y\right\rangle=\left\langle\psi_{0}(T) P_{m} x, P_{m} y\right\rangle$. It follows that

$$
\psi_{0}\left(M_{0}^{\prime} \cap \operatorname{Alg}\left\{P_{n}: n \in \mathbf{Z}\right\}\right) \subseteq R \cap \operatorname{Alg}\left\{P_{n}: n \in \mathbf{Z}\right\}
$$


Suppose now that $T \in R$ and $S \in M_{0}^{\prime}$. Then, for $n \in \mathbf{Z}, \beta_{n}(T S)=T \beta_{n}(S)$ and, therefore $\left\langle\psi_{0}(T S) x, y\right\rangle=m(g)$, where $g(n)=\left\langle T \beta_{n}(S) x, y\right\rangle=\left\langle\beta_{n}(S) x, T^{*} y\right\rangle$. Thus $\psi_{0}(T S)=T \psi_{0}(S)$.

Note, however, that $\psi_{0}$ need not be an expectation since we might have $\psi_{0}(I) \neq I$ (one can easily find examples where $\operatorname{sp}(\alpha)$ is finite and then $\psi_{0}(I)=0$ since $\beta_{n}(I)=$ 0 for all but finitely many $n$ 's). As $I \in \operatorname{Alg}\left\{P_{n}\right\} \cap\left(\operatorname{Alg}\left\{P_{n}\right\}\right)^{*}, \psi_{0}(I) \in \operatorname{Alg}\left\{P_{n}\right\} \cap$ $\left(\operatorname{Alg}\left\{P_{n}\right\}\right)^{*}=\left\{P_{n}: n \in \mathbf{Z}\right\}^{\prime}$. Hence $\psi_{0}(I) \in R_{0}$. Also $\psi_{0}(I) T=\psi_{0}(T)=T \psi_{0}(I)$ for $T \in R$; hence $\psi_{0}(I) \in M \cap R_{0}=Z\left(M_{0}\right) \cap R_{0}$.

We now let $q_{i}$ be $f_{i}-f_{i+1}$ if $i \geq 0$ and $f_{i+1}-f_{i}$ if $i<0$. Then $I=\sum_{i=0}^{\infty} q_{i}+f_{+}=$ $\sum_{i=-\infty}^{-1} q_{i}+f_{-}$. For $i \geq 0, j<0$ let $q_{i j}$ be $q_{i} q_{j}$. Then

$$
\sum_{i \geq 0, j<0} q_{i j}=\sum_{i \geq 0} q_{i}\left(\sum_{j<0} q_{j}\right)=\sum_{i \geq 0} q_{i}\left(I-f_{-}\right)=\left(1-f_{+}\right)\left(1-f_{-}\right)=1-f_{\infty} .
$$

Also note that, for $i \geq 0, j<0$ and $n \in \mathbf{Z}$,

$$
q_{i j} f_{n}=\left(f_{i}-f_{i+1}\right)\left(f_{i+1}-f_{j}\right) f_{n}= \begin{cases}0 & \text { if } n>i \text { or } n \leq j \\ q_{i j} & \text { if } j<n \leq i\end{cases}
$$

Now, for fixed $i \geq 0, j<0$ and $x, y \in H,\left\langle q_{i j} \psi_{0}(I) x, y\right\rangle=m(g)$, where $g(n)=$ $\left\langle q_{i j} f_{n} x, y\right\rangle$. But

$$
\frac{1}{2 N+1} \sum_{k=-N}^{N}\left\langle q_{i j} f_{k} x, y\right\rangle=\frac{i-j}{2 N+1}\left\langle q_{i j} x, y\right\rangle \rightarrow 0 \quad \text { as } N \rightarrow \infty .
$$

Hence $\left\langle q_{i j} \psi_{0}(I) x, y\right\rangle=m(g)=0$. This holds for all $x, y$ and, therefore, $q_{i j} \psi_{0}(I)=0$ for each $i \geq 0, j<0$. Summing over $i, j$ we find that $\left(1-f_{\infty}\right) \psi_{0}(I)=0$. Hence $\psi_{0}(I)=f_{\infty} \psi_{0}(I)$.

For $x \in H$ we have $\left\langle\psi_{0}(I) f_{+} x, x\right\rangle=m(g)$, where $g(n)=\left\langle f_{n} f_{+} x, x\right\rangle$. For $n \geq 0$, $g(n)=\left\langle f_{+} x, x\right\rangle$ and, therefore

$$
\begin{aligned}
g^{\prime}(n) & =\frac{1}{2 N+1} \sum_{n=-N}^{N}\left\langle f_{n} f_{+} x, x\right\rangle \\
& \geq \frac{1}{2 N+1}(N+1)\left\langle f_{+} x, x\right\rangle \rightarrow \frac{1}{2}\left\langle f_{+} x, x\right\rangle \quad \text { as } N \rightarrow \infty
\end{aligned}
$$

Thus $\left\langle\psi_{0}(I) f_{+} x, x\right\rangle \geq \frac{1}{2}\left\langle f_{+} x, x\right\rangle$. Hence $\psi_{0}(I) f_{+} \geq \frac{1}{2} f_{+}$. Similarly $\psi_{0}(I) f_{-} \geq \frac{1}{2} f_{-}$ and consequently $f_{\infty} \psi_{0}(I) \geq \frac{1}{2} f_{\infty}$. In particular $f_{\infty}$ is the range projection of $\psi_{0}(I)$ (hence lies in $\left.R_{0}\right)$. Therefore there is some $h \in Z\left(M_{0}\right) \cap R_{0}$ such that $h \psi_{0}(I)=f_{\infty}$ (and $h \geq 0$ ).

Let $\bar{\psi}_{1}(T)$ be $h \psi_{0}(T)$ for each $T \in M_{0}^{\prime}$. Then $\psi_{1}$ has the following properties (which follow from the properties of $\psi_{0}$ and $h$ ):

(1) $\psi_{1}$ is a linear, positive map from $M_{0}^{\prime}$ into $R$ (note: $\psi_{1}(T)=h^{1 / 2} \psi_{0}(T) h^{1 / 2}$ ).

(2) $\psi_{1}(S T)=\psi_{1}(S) T$ for $T \in R, S \in M_{0}^{\prime}$.

(3) $\psi_{1}$ maps $M_{0}^{\prime} \cap \operatorname{Alg}\left\{P_{n}: n \in \mathbf{Z}\right\}$ into $R \cap \operatorname{Alg}\left\{P_{n}: n \in \mathbf{Z}\right\}$.

(4) $\psi_{1}(T)=f_{\infty} T$ for $T \in R$.

Next we construct another map, $\psi_{2}$, from $M_{0}^{\prime}$ into $R$ with properties (1)-(3) as above (with $\psi_{1}$ replaced by $\psi_{2}$ ) and

$\left(4^{\prime}\right) \psi_{2}(T)=\left(1-f_{\infty}\right) T$ for $T \in R$. 
The sum $\psi_{1}+\psi_{2}$ is the required expectation from $M_{0}^{\prime}$ into $R$.

Now fix $T \in M_{0}^{\prime}, i \geq 0$ and $j<0$. Let $T_{i j}$ be $(1 /(i-j)) \sum_{n=-\infty}^{\infty} \beta_{n}(T) q_{i j}$. By (*) the sum has at most $i-j$ nonzero terms and it defines an operator $T_{i j} \in q_{i j} M_{0}^{\prime} q_{i j}$ (note that $\beta_{n}(T) q_{i j}=q_{i j} \beta_{n}(T)$ ). Define $\psi_{2}(T)$ to be $\psi_{2}(T)=\sum_{i \geq 0, j<0} T_{i j}$. As $\left\{q_{i j}: i \geq 0, j<0\right\}$ is an orthogonal family of projections, and $T_{i j} \stackrel{\in}{\in} q_{i j} M_{0}^{\prime} q_{i j}$, $\psi_{2}(T)$ is a well-defined operator in $M_{0}^{\prime}$ and $\psi_{2}(T)=\left(1-f_{\infty}\right) \psi_{2}(T)\left(1-f_{\infty}\right)$ (since $\left.1-f_{\infty}=\sum q_{i j}\right)$.

For $m \in \mathbf{Z}, T \in M_{0}^{\prime}, i \geq 0$ and $j<0$ we have

$$
\beta_{m}\left(T_{i j}\right)=\frac{1}{i-j} \sum_{n=-\infty}^{\infty} \beta_{m} \beta_{n}(T) \beta_{m}\left(q_{i j}\right)=f_{m} \frac{1}{i-j} \sum_{n} \beta_{n+m}(T) \beta_{m}\left(q_{i j}\right)
$$

But

$$
\begin{aligned}
\beta_{m}\left(q_{i j}\right) & =\beta_{m}\left(q_{i} q_{j}\right)=\beta_{m}\left(\left(f_{i}-f_{i+1}\right)\left(f_{j+1}-f_{j}\right)\right) \\
& =f_{m}\left(f_{i+m}-f_{i+m+1}\right)\left(f_{j+m+1}-f_{j+m}\right) \\
& = \begin{cases}f_{m} q_{i+m, j+m} & \text { if } i+m \geq 0, j+m<0 \\
0 & \text { otherwise. }\end{cases}
\end{aligned}
$$

Hence, for $-i \leq m<-j$,

$$
\beta_{m}\left(T_{i j}\right)=f_{m} \frac{1}{i-j} \sum_{n} \beta_{m+n}(T) q_{i+m, j+m}=f_{m} T_{i+m, j+m}
$$

and for $m \geq-j$ or $m<-i, \beta_{m}\left(T_{i j}\right)=0$.

Suppose that $m>0$. Then (by $(*)) f_{m} q_{i+m, j+m}=0$ if $-m \leq i<0$ and, therefore,

$$
\begin{aligned}
\beta_{m}\left(\psi_{2}(T)\right) & =\sum_{i \geq 0, j<0} f_{m} T_{i+m, j+m} \\
& =\sum_{i+m \geq 0, j+m<0} f_{m} T_{i+m, j+m}=f_{m} \psi_{2}(T) .
\end{aligned}
$$

Similarly $\beta_{m}\left(\psi_{2}(T)\right)=f_{m} \psi_{2}(T)$ for $m<0$. Hence $\psi_{2}(T) \in R$ for each $T \in M_{0}^{\prime}$.

Suppose now that $T \in M_{0}^{\prime} \cap \operatorname{Alg}\left\{P_{n}: n \in \mathbf{Z}\right\}$. Then, for $i \geq 0, j<0, T_{i j} \in$ $\operatorname{Alg}\left\{P_{n}: n \in \mathbf{Z}\right\}$ (since, as was shown before, $\beta_{m}(T) \in \operatorname{Alg}\left\{P_{n}: n \in \mathbf{Z}\right\}$ for each $m \in \mathbf{Z}$ and $\left.q_{i j} \in M_{0}\right)$. Hence $\psi_{2}\left(M_{0}^{\prime} \cap \operatorname{Alg}\left\{P_{n}: n \in \mathbf{Z}\right\}\right) \subseteq R \cap \operatorname{Alg}\left\{P_{n}: n \in \mathbf{Z}\right\}$.

For $T \in R, \beta_{N}(T)=f_{n} T$ for each $n \in \mathbf{Z}$. Hence

$$
T_{i j}=\frac{1}{i-j} \sum_{n} f_{n} T q_{i j}=\frac{1}{i-j} \sum_{n} f_{n} q_{i j} T
$$

for each $i \geq 0, j<0$. Using $(*)$,

$$
T_{i j}=\frac{1}{i-j} \sum_{n=j+1}^{i} q_{i j} T=q_{i j} T \text {. }
$$

Hence $\psi_{2}(T)=\left(1-f_{\infty}\right) T$.

Therefore $\psi_{2}$ satisfies the following properties:

$\left(1^{\prime}\right) \psi_{2}$ is a positive linear map from $M_{0}^{\prime}$ into $R$. 
$\left(2^{\prime}\right) \psi_{2}(S T)=\psi_{3}(S) T$ for $S \in M_{0}^{\prime}, T \in R$ as

$\psi_{2}(S T) q_{i j}=(1-j)^{-1} \sum_{n} \beta_{n}(S T) q_{i j}=(1-j)^{-1} \sum \beta_{n}(S) T q_{i j}=\psi_{2}(S) T q_{i j}$.

$\left(3^{\prime}\right) \psi_{2}\left(M_{0}^{\prime} \cap \operatorname{Alg}\left(P_{n}: n \in \mathbf{Z}\right\}\right) \subseteq R \cap \operatorname{Alg}\left\{P_{n}: n \in \mathbf{Z}\right\}$

$\left(4^{\prime}\right) \psi_{2}(T)=\left(1-f_{\infty}\right) T$ for $T \in R$.

Then $\psi=\psi_{1}+\psi_{2}$ is the required expectation.

Suppose $R_{0}^{\prime}$ is an injective von Neumann algebra. Then there is an expectation $\phi$ from $B(H)$ onto $R_{0}^{\prime}$. Since $\left\{P_{n}: n \in \mathbf{Z}\right\} \subseteq R_{0}^{\prime}, \phi$ maps $\operatorname{Alg}\left\{P_{n}: n \in \mathbf{Z}\right\}$ onto $R_{0}^{\prime} \cap \operatorname{Alg}\left\{P_{n}: n \in \mathbf{Z}\right\}$. Let $\phi_{0}$ be the map $\psi^{\prime} \circ \phi$ (where $\psi^{\prime}$ is the expectation from $R_{0}^{\prime}$ onto $M$ whose existence was proved in Theorem 4.6). Then $\phi_{0}$ is an expectation onto $M$ and $\phi_{0}\left(\operatorname{Alg}\left\{P_{n}: n \in \mathbf{Z}\right\}\right)=H^{\infty}(\alpha)$.

COROLlary 4.7. Suppose $R_{0}^{\prime}$ is injective. Then, for $a \in M, \operatorname{dist}\left(a, H^{\infty}(\alpha)\right)=$ $\left\|\left(1-P_{0}\right) a P_{0}\right\|$. (Here $\operatorname{dist}\left(a, H^{\infty}(\alpha)\right)$ is $\inf \left\{\|a-b\|: b \in H^{\infty}(\alpha)\right\}$.)

ProOF. As Arveson shows in [3], for any algebra $A(\subseteq B(H))$ and operator $a \in B(H), \operatorname{dist}(a, \mathcal{A}) \geq \operatorname{Sup}\{\|(1-P) a P\|: P \in$ Lat $\mathcal{A}\}$ (where Lat $=\{P: P$ is a projection such that $P b P=b P$ for all $b \in A\})$. Hence

$$
\begin{aligned}
& \left\|\left(1-P_{0}\right) a P_{0}\right\| \leq \operatorname{Sup}\left\{\left\|\left(1-P_{n}\right) a P_{n}\right\|: n \in \mathbf{Z}\right\} \\
& \leq \operatorname{Sup}\left\{\|(1-P) a P\|: P \in \operatorname{Lat} H^{\infty}(\alpha)\right\} \leq \operatorname{dist}\left(a, H^{\infty}(\alpha)\right) \quad \text { for } a \in M .
\end{aligned}
$$

It will suffice, therefore, to show

$$
\left\|\left(1-P_{0}\right) a P_{0}\right\| \geq \operatorname{Sup}\left\{\left\|\left(1-P_{n}\right) a P_{n}\right\|: n \in \mathbf{Z}\right\} \geq \operatorname{dist}\left(a, H^{\infty}(\alpha)\right) .
$$

Let $\phi_{0}$ be the expectation from $B(H)$ onto $M$ as in the discussion preceding this corollary. By Arveson's distance formula [3],

$$
\operatorname{dist}\left(a, \operatorname{Alg}\left\{P_{n}: n \in \mathbf{Z}\right\}\right)=\operatorname{Sup}\left\{\left\|\left(1-P_{n}\right) a P_{n}\right\|: n \in \mathbf{Z}\right\} .
$$

Let us write $t$ for the right-hand side of this equation and, for $\varepsilon>0$, we can find $a_{\varepsilon} \in \operatorname{Alg}\left\{P_{n}: n \in \mathbf{Z}\right\}$ such that $\left\|a-a_{\varepsilon}\right\| \leq t+\varepsilon$. Applying $\phi_{0}$ we get $\left\|a-\phi_{0}\left(a_{\varepsilon}\right)\right\|=\left\|\phi_{0}\left(a-a_{\varepsilon}\right)\right\| \leq\left\|a-a_{\varepsilon}\right\| \leq t+\varepsilon$. As $\phi_{0}\left(a_{\varepsilon}\right) \in H^{\infty}(\alpha)$ and, the choice of $\varepsilon>0$ is arbitrary, we have $\operatorname{dist}\left(a, H^{\infty}(\alpha)\right) \leq t$.

Now fix $\varepsilon>0$. Then there are $n \in \mathbf{Z}$ and $x \in P_{n}(H)$ such that $\left\|\left(1-P_{n}\right) a x\right\|>$ $t-\varepsilon$ and $\|x\| \leq 1$. Let $F$ be the orthogonal projection onto $\left[H^{\infty}(\alpha) x\right]$. Then $F \leq P_{n}$ and $t-\varepsilon<\left\|\left(1-P_{n}\right) a x\right\| \leq\|(1-F) a F x\|$.

By Corollary 3.6 there is a partial isometry $U \in R$ such that $U^{*} U \in R_{0}$ and $F=U P_{0} U^{*}$ (since $\left.F \leq P_{n}, F \in P_{3}\right)$. Hence

$$
\begin{aligned}
t-\varepsilon & <\|(1-F) a F x\|=\left\|\left(1-U P_{0} U^{*}\right) a U P_{0} U^{*} x\right\|=\left\|U\left(1-P_{0}\right) U^{*} a U P_{0} U^{*} x\right\| \\
& =\left\|U\left(1-P_{0}\right) a P_{0} U^{*} x\right\| \leq\left\|\left(1-P_{0}\right) a P_{0}\right\| .
\end{aligned}
$$

Since $\varepsilon>0$ was arbitrary, $t \leq\left\|\left(1-P_{0}\right) a P_{0}\right\|$.

COROllary 4.8. Suppose $R_{0}^{\prime}$ is injective. Let $\left\{a_{i}: 1 \leq i \leq N\right\}$ in $H^{\infty}(\alpha)$ satisfy, for some $\varepsilon>0$,

$$
\sum_{i=1}^{N}\left\|\left(1-P_{n}\right) a_{i} x\right\|^{2} \geq \varepsilon^{2}\left\|\left(1-P_{n}\right) x\right\|^{2}
$$


for all $n \in \mathbf{Z}, x \in H$. Then there are $\left\{b_{i}: 1 \leq i \leq N\right\} \subseteq H^{\infty}(\alpha)$ (that can be chosen so that $\left\|b_{i}\right\| \leq 4 N \varepsilon^{-3}$ if $\left\|a_{i}\right\| \leq 1$ for each $\left.1 \leq i \leq N\right)$ such that $\sum_{i=1}^{N} b_{i} a_{i}=I$.

ProOF. Consider $\left\{a_{i}\right\}_{i=1}^{N}$ as elements of $\operatorname{Alg}\left\{P_{n}: n \in \mathbf{Z}\right\}$. Then by [3, Theorem 4.3] there are $\left\{b_{i}^{\prime}\right\}_{i=1}^{N}$ (with $\left\|b_{i}^{\prime}\right\| \leq 4 N \varepsilon^{-3}$ if $\left\|a_{i}\right\| \leq 1$ for each $1 \leq i \leq N$ ) such that $\sum b_{i}^{\prime} a_{i}=I$ and $\left\{b_{i}^{\prime}\right\}_{i=1}^{N} \subseteq \operatorname{Alg}\left\{P_{n}: n \in \mathbf{Z}\right\}$. Applying the expectation $\phi_{0}$ (from $B(H)$ onto $M$, as in the discussion preceding Corollary 4.7) we get $\sum \phi_{0}\left(b_{i}^{\prime}\right) a_{i}=I$ and $\left\|\phi_{0}\left(b_{i}^{\prime}\right)\right\| \leq\left\|b_{i}^{\prime}\right\|$. Let $b_{i}$ be $\phi_{0}\left(b_{i}^{\prime}\right)$ and we are done.

REMARK. Note that $R_{0}^{\prime}$ is injective if and only if $M$ is. Indeed, if $M$ is injective then so is $R$. But $R_{0}=\varepsilon_{0}(R)$ and consequently $R_{0}$ and $R_{0}^{\prime}$ are injective von Neumann algebras. On the other hand if $R_{0}^{\prime}$ is injective, then it follows from Theorem 4.6 that $M$ is injective too.

\section{REFERENCES}

1. W. B. Arveson, Analyticity in operator algebras, Amer. J. Math. 89 (1967), 578-642.

2. - On groups of automorphisms of operator algebras, J. Funct. Anal. 15 (1974), 217-243.

3. __ Interpolation problems in nest algebras, J. Funct. Anal. 20 (1975), 208-233.

4. J. Dixmier, Von Neumann algebras, North-Holland, Amsterdam, 1981.

5. S. Kawamura and J. Tomiyama, On subdiagonal algebras associated with flows in operator algebras, J. Math. Soc. Japan 29 (1977), 73-90.

6. R. I. Loebl and P. S. Muhly, Analyticity and flows in von Neumann algebras, J. Funct. Anal. 29 (1978), 214-252.

7. M. McAsey, P. S. Muhly and K.-S. Saito, Nonselfadjoint crossed products (invariant subspaces and maximality), Trans. Amer. Math. Soc. 248 (1979), 381-409.

8. __ Nonselfadjoint crossed products. II, J. Math. Soc. Japan 33 (1981), 485-495.

9. K.-S. Saito, The Hardy spaces associated with a periodic flow on a von Neumann algebra, Tôhoku Math. J. 29 (1977), 69-75.

10. __ Invariant subspaces for finite maximal subdiagonal algebras, Pacific J. Math. 93 (1981), 431434.

11. , Nonselfadjoint subalgebras by compact abelian actions on finite von Neumann algebras, Tôhoku Math. J. 34 (1982), 485-494.

12. __ Spectral resolutions of invariant subspaces by compact abelian group actions on von Neumann algebras, preprint.

13. B. Solel, The invariant subspace structure of nonselfadjoint crossed products, Trans. Amer. Math. Soc. 279 (1983), 825-840.

14. __ Invariant subspaces for algebras of analytic operators associated with a periodic flow on a finite von Neumann algebra, preprint.

15. ___ Algebras of analytic operators associated with a periodic flow on a von Neumann algebra, preprint.

Department of Mathematics, Statistics and Computing Science, Dälhousie University, Halifax B3H 4H8, Nova Scotia, CANADA 